\title{
Design and Analysis of Truss Type Steel Buliding by using Tekla Software
}

\author{
G.Venkata Rao ${ }^{1}$ A.Sai Kumar ${ }^{2}$ | Dr. Dumpa Venkateswarlu ${ }^{3}$ \\ ${ }^{1}$ PG Scholar, Department of Civil Engineering, Godavari Institute of Engineering \& Technology (A), Rajahmundry \\ ${ }^{2}$ Assistant Professor, Department of Civil Engineering, Godavari Institute of Engineering \& Technology (A), Rajahmundry \\ ${ }^{3}$ Professor \& Head, Department of Civil Engineering, Godavari Institute of Engineering \& Technology (A), Rajahmundry
}

\section{To Cite this Article}

G.Venkata Rao, A.Sai Kumar and Dr. Dumpa Venkateswarlu, "Design and Analysis of Truss Type Steel Buliding by using Tekla Software", International Journal for Modern Trends in Science and Technology, Vol. 07, Issue 03, March 2021, pp.: 256-271.

\section{Article Info}

Received on 15-February-2021, Revised on 10-March-2021, Accepted on 21-March-2021, Published on 23-March-2021.

\section{ABSTRACT}

Steel offer the range of advantages to the structure Industry. Steel is also one of the most sustainable construction materials, building owners naturally value the flexibility of steel buildings in addition the value of benefits they provide. Steel trusses are widely used to carry the roof loads and to provide horizontal stability. There are numerous advantages to using steel trusses instead of traditional wood trusses, but the main reasons are simplicity and strength. Steel trusses offer a high strength, light weight roof system that can be installed quickly. In this project I use Tekla software. It has a very interactive user interface which allows the users to draw the frame and input the load values and dimensions. Tekla structures are powerful and flexible software for all structural projects. Then according to the specified criteria assigned it analyses the structure and designs the members with structural steel.Our final work was the proper analysis and design of truss type steel building. The Aim of present study is to define proper technique for creating Geometry, cross sections for column and beam etc., developing specification and supports conditions, types of Loads and load combinations. I analyzed and designed a truss type steel building initially for all possible load combinations (dead, live, wind, seismic loads). In this analysis process different types of codes are utilized. Dead load IS:875(Part-1), Live load IS: 875(Part-2), wind load IS 875-(Part-3), seismic load IS 1893. In this study a truss type steel structure is analyzed for seismic and wind load combination using tekla. Implemented manual designing and modelling by using of Tekla software

KEYWORDS: Design, Analysis, Geometry, Structure, Wind load, seismic, Tekla Structures.

\section{INTRODUCTION}

A Truss is an important structure type in structural engineering. They are one of the most widely adopted structural designs, many times being utilized as the structural solution of choice for bridges, roofs, cranes, aircrafts, and even robots and spaceships. A steel truss is an arrangement of steel pieces connected to form a structure intended to span or bridge a gap of some sort. A steel truss transfers weight, or pressure applied to it, to weight-bearing structures on either side of the gap. Steels trusses come in many types and are commonly used for large roofs and bridges. While any truss can be made of steel to improve its load-carrying capabilities, and many often are, there are several types of steel truss that are more common than others.

Trusses are used in a broad range of buildings, mainly where there is a requirement for very long spans, such as in airport terminals, aircraft hangers, sports stadia roofs, 
auditoriums and other leisure buildings. Trusses are also used to carry heavy loads and are sometimes used as transfer structures. There are many types of trusses available for residential, commercial and industrial building construction. Each type has specific design considerations and advantages and disadvantages

\section{Advantages of structural steel buliding:}

The use of steel building as industrial building is growing fast in all parts of the world. These buildings are typically used for workshops, factories, industries and distribution warehouses. Steel buildings in general have many advantages such as

- The high quality of construction.

- Lower maintenance cost.

- Non-combustible to fire.

- Environment friendly.

- Steel components can be used again.

- Faster than any construction method.

\section{Disadvantages of structural steel buliding:}

Steel buildings have also disadvantaged such as

- The change of steel cost from time to time, subject to corrosion.

- Susceptibility to buckling.

- Less availability of steel.
$>$ This project includes the analysis, design and detailing of structures like a beams, columns and other structural components.

$>$ To create the 3D models of the structure by using the Tekla software.

$>$ Analyzed and designed a truss type steel building initially for all possible load combinations

\section{LITERATURE REVIEW}

\section{MA Shan-yu}

This paper illustrate On comprehensive analysis of application of steel structure's detailing design software Tekla Structure .The paper introduces the domestic background of the steel structure's detailing design software Tekla Structure, illustrates the current situation of the software in country's application, indicates all kinds of advantages functions of the software, analyzes the advantages and disadvantages of the software in the project application, and explores the methods to improve the software's application efficiency in projects, so as to find the best way to exert the software's advantages.

\section{JIANG Wen-ya (China Petroleum First Construction Co. Luoyang 471023, China),MA Xiu-juan}

Has done research work on Modeling of Special-shaped Structure Using Software Tekla structures. In recent years, with the substantial .development in the national economy, applications tof steel structures have widely increased. The needs for architectural appearance are increased too. Combined with the practical applications of the software Tekla Structures in detailed designs of irregular steel structures, this paper discusses the key skills in using the software Tekla Structures for special-shaped structure modeling with respect to modeling and plotting, as well as the significance to field steel structures construction.

members, aesthetics and any necessity to connect fi spans, tee sections are frequently used for chords with with angles used as internal members. The internah This paper illustrate "Modelling conception of members may be bolted or welded to the tees. sustainable steel building by means of Tekla Back-to-back angles or channels may be used for software" The mainly important element governing longer spans or heavier loads, with a gusset plate the option of steel (I-section)\& appearance of used at nodes to connect the members

\subsection{Objectives of the Study}

$>$ The main objective of the project is to analyze and design of truss type steel building . construction designed for any constituent is its structural integrity. while high specific strength as well as well intended project with Tekla software. It is used to choose the steel I-Sections for strength and durability of the building to accept a range of type of dead loads, live loads and wind loads. 
Development for energy efficiency, water efficiency and to get better the indoor environment

\section{L.Vimala, T.Naresh Kumar, S.M.V.Narayana, J.Chinna Babu}

This paper illustrates an Assessment and Design of Steel frame Structure, consists Performance of Connection Joints with Tekla \& Staad Pro. In connection with the handling of structures it is noticed that steel is the simplest material to model since it is isotropic. The main objective of this paper compared to without bracings, with bracings is the more effective in different loads and conditions. Horizontal bracings are used as alternative techniques. By using horizontal and vertical bracings displacement, moment, and shear force came to reduce. Results clearly shows huge difference of with bracings and without bracings

K.K.Sangle, K.M.Bajori, V.Mhalungkar (2012) Has done research work on "Seismic Analysis Of High Rise Steel Frame Building With And With Out Bracing" The Aim of study was to compare the results of seismic analysis of high rise steel building with different pattern of bracing system and without bracing system. By using time history analysis the result of the study shows that bracing element will have very important effect on structural behavior under earthquake effect.

ValeriiaLobanova. et.al (2017)The aspire of this thesis was "Comparison of structural modelling in open BIM projects". Tekla Structures as well as Revit, in order to recognize difference in open BIM IFC-models according to general BIM requirements 2012 and recover out benefits of modelling in every program. This theme is definite, as at the moment more consumers need information models of buildings, which hold all information concerning the facility.

\section{III.DESCRIPTION OF THE PROJECT}

\section{Structural design}

Structural design might have a complicated process with a number of steps and instruments. Material experts, BIM, fire and structural engineers and a number of other professionals might be involved. Figure 1 below shows the flow chart of a typical structural design process. Structural design means artistic invention and dimensioning. Invention is the creation of a structural form, dimensioning is to assign to every structural member adequate dimensions for stability, serviceability, suitability and sustainability

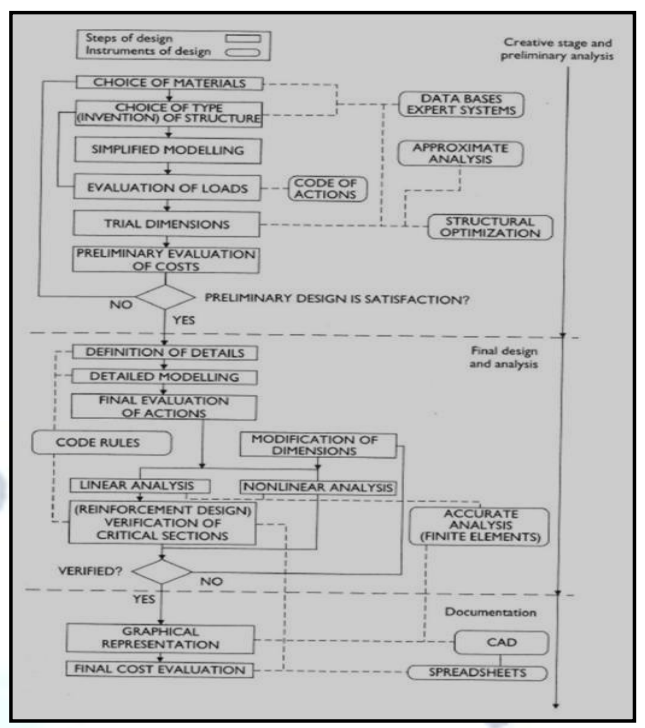

Figure 1. Flow-chart of the structural design process

. Structural analysis alone was conducted. There was no need to additionally design and change the dimensions of the structural components since the requirement was to use the structures of certain profiles. Nevertheless, the design resistance of structural members is checked and verified. Possible changes are considered.

The truss type steel building project consisted of the following steps:

- Defining the structural solutions such as trusses and bracing

- Calculating the loads acting on the structure

- Performing structural analysis in Tekla Structural Design

- Design resistance check of structural connections

- Building a BIM model in Tekla Structures

- Making shop drawings for production

\section{Codes and standards}

Steel design codes in the 1930's were working stress codes in which the member stresses determined by analyzing the structure under the working loads were required to be less than the permissible working stresses set out in the codes. There are no Indian standards codes as such for the control of corrosion. The latest editions of the following organizations' standards, codes, and guidelines shall be used for the design of corrosion control systems. This chapter covers the applicable codes and standards for the analysis, design and detailing of truss type steel building.

Structural calculations in this project are based on the following standards: 
$>$ IS: 1893(Part-1) - 2002, Criteria for Earthquake Resistant Design of Structures: General provisions and Buildings.

$>$ IS: 875 (Part-1\&2)-1987, Code Of Practice for Design Loads (Other Than Earthquakes) For Buildings \& Structures - Imposed loads.

$>$ IS: 875 (Part-3)-1987, Code Of Practice for Design Loads (Other Than Earthquakes) For Buildings \& Structures - Wind loads.

$>$ IS: 456-2000, Code Of Practice For Plain \& Reinforced Concrete

$>$ IS 13920: 1993 - Indian Standard - Ductile Detailing of Reinforced Concrete Structures Subjected to Seismic Forces - Code of practice.

\section{Software used during the project Tekla structures}

Tekla Structures is building information modeling software that is able to create structures and to store information on the components of the building. The software allows designers to make 3D models of structures as well as produce technical drawings and make calculations on the amount of materials needed for construction. Figure 2 below shows the license information of the software. Tekla Structures is 3D building information modeling (BIM) software used in the building and construction industries for steel and concrete detailing, precast and cast in-situ. The software enables users to create and manage 3D structural models in concrete or steel, and guides them through the process from concept to fabrication.

The process of shop drawing creation is automated.

\section{Tekla structural designer $2019 i$}

- A BIM Software from Trimble software Co-operation

- Analysis and design buildings efficiently

- Fully automated and packed with many unique features of optimized concrete and steel design

- Design a building structure and its components using 3D modelling, generate 2D drawings and access building information.

- Often used in conjunction with Autodesk Revit, where structural framing is designed in Tekla and exported to Revit using the DWG/DXF formats Analysis and Design of truss type steel buliding Using Tekla

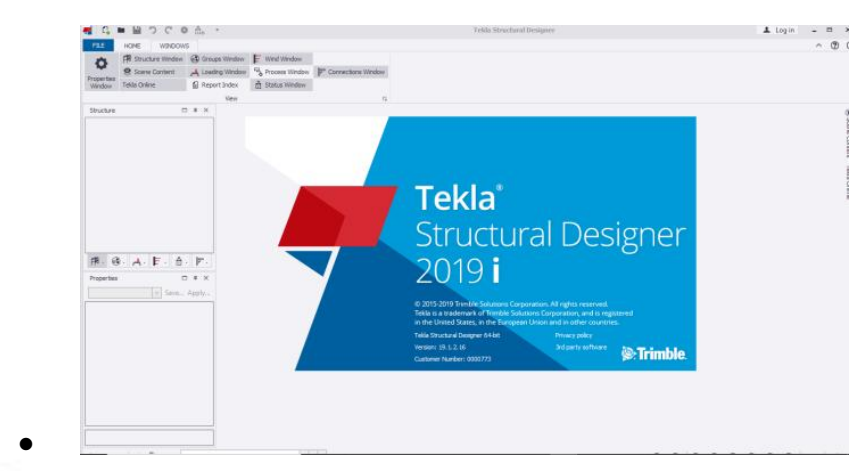

Figure 2: Tekla Structural Designer license window

\section{AutoCAD 2016}

- AutoCAD is a commercial computer aided design (CAD) and drafting software application.

- Developed and marketed by Autodesk, AutoCAD was first released in December 1982

- $\quad$ Since 2010, Autocad was released as a mobile and web app as well, marketed as AutoCAD 360 .

\section{IV.STRUCTURAL BUILDING COMPONENTS}

uctural building components are described in this part of the report. The choice of material was limited because one of the client's demands.Profiles of structural components together with their steel grades used in the project are presented in Table 1. 


\begin{tabular}{|ccc|}
\hline $\begin{array}{c}\text { Structural } \\
\text { component }\end{array}$ & Profiles & $\begin{array}{r}\text { Steel } \\
\text { grade }\end{array}$ \\
\hline $\begin{array}{c}\text { Load-bearing } \\
\text { columns }\end{array}$ & SHS100x100x5 & S355 \\
\hline Horizontal beams & SHS60x60x4 & S355 \\
\hline $\begin{array}{c}\text { Beams at the } \\
\text { ends of the roof } \\
\text { structure }\end{array}$ & SHS100x100x5 & S355 \\
\hline $\begin{array}{c}\text { Bracing } \\
\text { Top and bottom } \\
\text { chords of the } \\
\text { trusses }\end{array}$ & SHS60x60x4 & S355 \\
\hline Truss webs & SHS60x60x4 & S355 \\
\hline
\end{tabular}

Table 1. Profiles and steel grade of the structural building components

\section{Foundation}

Even though the foundation is not in the scope of this report, it is important to know the initial dimensions. This information is needed to understand the support conditions of the structure and later to calculate the resistance of the column-base connection.

The foundation in a building serves for transferring loads from the superstructure to the ground. Load-bearing columns that transfer loads from the structure are placed on top of $200 \mathrm{~mm}$ concrete foundation.

The foundation transfers loads further to the footing. Figure 22 below shows the preliminary design of the

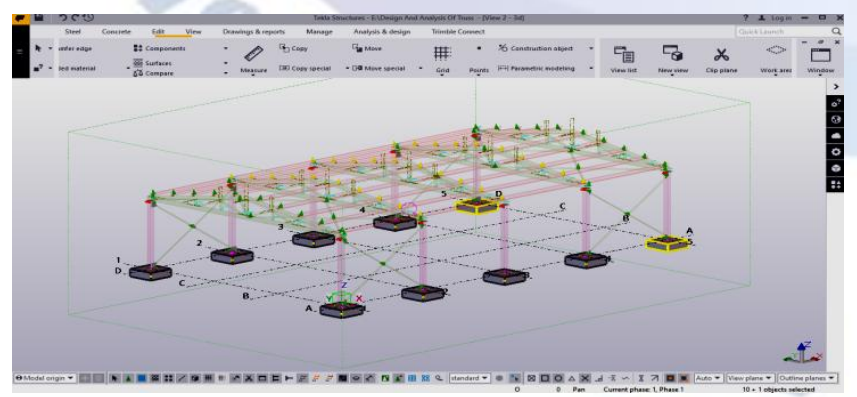

foundations provided by the client

Figure3: Foundation modeled using Tekla Structures

\section{Columns}

There is a total of 17 load-bearing columns in the structure. Columns that are non-load bearing are used in door frames and are not considered in the calculations. Figure 4 demonstrates the placement of the columns in the structure.

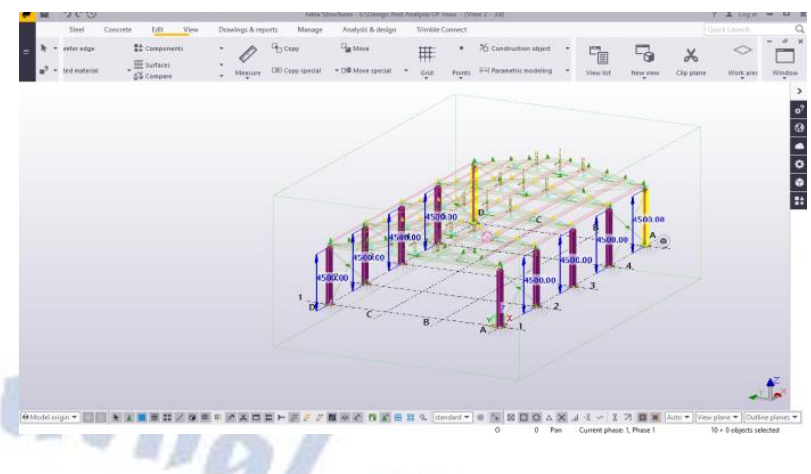

Figure 4: Columns modeled using Tekla Structures

\section{Roof bracing}

Since the dimensions of the foundation do not allow to make proper moment-resisting connections, it is decided to design a roof structure that will take most of the horizontal loads. Triangulation method is used in the design of the structure..

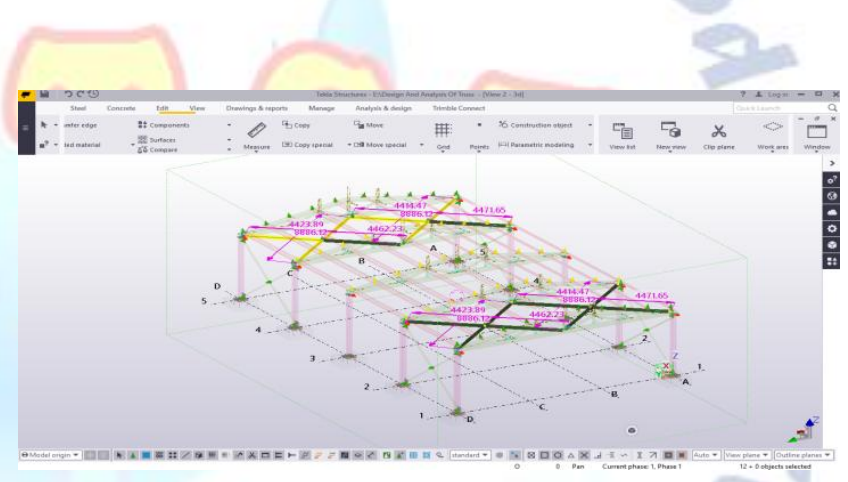

Figure 5. Roof bracing modelled in Tekla Structures

It is ensured that the load paths are established and there is as less eccentricity created in members as possible. Figure 24 shows how the roof structure is designed.

\section{General geometry For Trusses}

For efficient structural performance, the ratio of span to truss depth should be chosen in the range 10 to 15 . The architectural design of the building determines its external geometry and governs the slope(s) given to the top chord of the truss. The intended use of the internal space can lead either to the choice of a horizontal bottom chord, e.g. where conveyors must be hung under the chord, or to an inclined bottom chord, to allow maximum space to be provided. For an efficient layout of the truss members between the chords, the following is advisable:The inclination of the diagonal members in relation to the chords should be between $35^{\circ}$ and $55^{\circ}$ Point loads should only be applied at nodes. 


\section{Geometry of the structure}

The analysis was done for a three-dimensional structure. The shape and dimensions of the structure are based on the model built in Tekla Structures. Here we are Provide different levels like eave, Ridge, Base levels. The Following Figure 6 Shows Different levels in Tekla structural Designer.

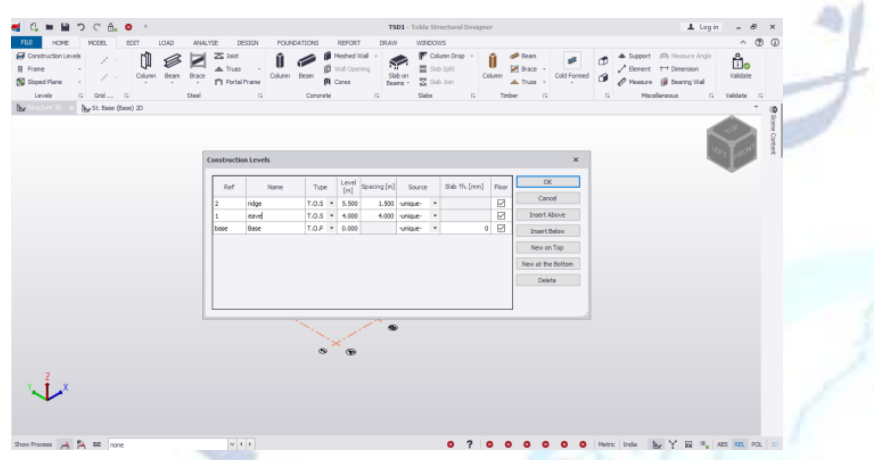

Figure 6.Shows Different levels in TSD software.

The structure consists of lines that are connected with nodes. Each line contains information on the member it represents. Members have beginnings and ends that help to define the releases. Figure 7 below illustrates the structural model built in TEKLA STRUCTURES

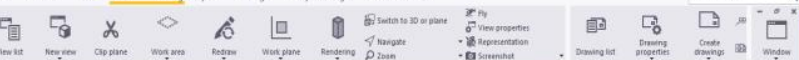

\section{Figure 7. Three-dimensional structure modeled in Têkla Structures}

Element Properties

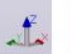

Table 2: List of Section Properties

\section{Modification Factors}

Table 3: List of Modification Factors

\section{METHODOLOGY}

The theme of this project remains towards assessment the whole building with different load combinations in addition to design of structure in line for towards custom of steel for instance a material. Then the consequence of essential steel building reaction now various conditions.

- Dead load on the structure
- Live load on the structure

- Wind load on the structure

- Seismic load on the structure

- Snow load of strucutre

Uncertainty the loads be there in sync besides taken complex at that moment economy remains affected. If economy stands reflected in addition loads remain taken reduced before the security remains compromised. Subsequently the approximation of a number of loads acting remains towards intended accurately. Indian Standard Code IS: 875-2007.

\section{Dead load on the structure:}

Dead load remains taken by means of recommend by IS:875-1987 (part-1) code of preparation design of loads (further seismic activity) intended for buildings in addition construction.

Self-weight of the structure is considered as the dead load acting on the structure. Since the load calculations were done before the roof truss structure was designed, it was decided to calculate the weight of the roof with an estimated value $\sigma=$ $5.8 \mathrm{~kg} / \mathrm{m}$ and the rest of the structure based on the Tekla model.

\section{Live load on the structure:}

Live load remains occupied as per prescribe by IS:875-1987 (part-2) code of practice for design of loads for buildings and structure. Live load is the action for which the variation in magnitude with time is neither negligible nor monotonic. This corresponds to loads related to the movement of people inside the building, movable furniture, etc. Load coming from the maintenance of the roof and load from the moving rail system that holds and transfers the carcass of an animal are considered

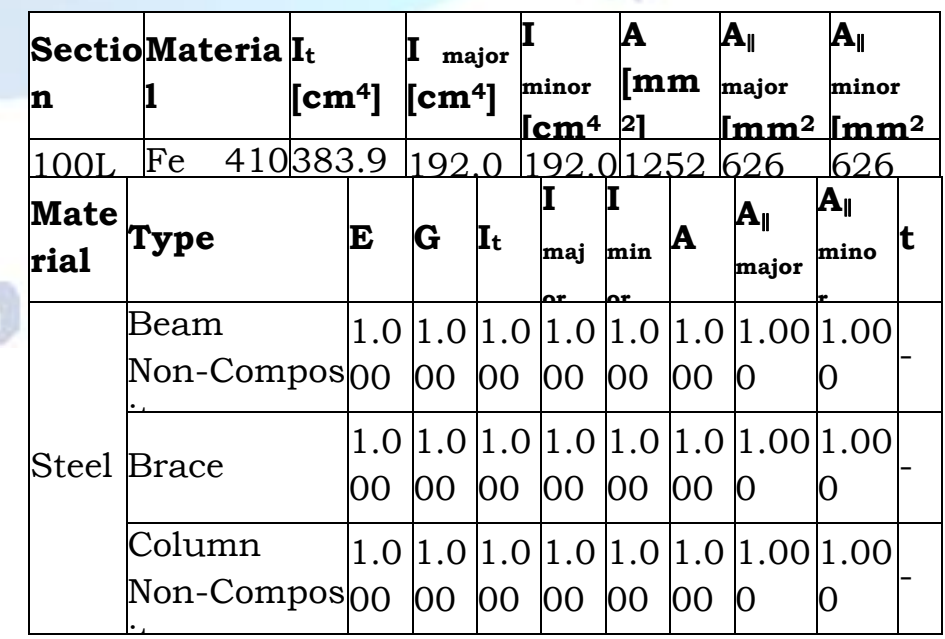

for live load calculations in the project 
The elementary wind speed intended on behalf of every site shall be there obtained from IS:875-1987 (part-3). It is $39 \mathrm{~m} / \mathrm{s}$ in addition shall be modified towards include the following effects towards get design wind velocity at any height $(\mathrm{Vz})$ for the chosen the structure. Risk level Terrain roughness, height in addition size of the structure, and level topography it be able to be mathematically expressed for example follows:

$\mathrm{Vz}=\mathrm{Vb} . \mathrm{k} 1 . \mathrm{k} 2 . \mathrm{k} 3$

$\mathrm{Vz}=$ design wind speed at any height $\mathrm{z}$ in $\mathrm{m} / \mathrm{s}$.

$\mathrm{k} 1$ =probability factor (risk coefficient) (Refer 5.3.1 of IS:875(Part 3 -1987))

k2 = terrain, height and structure size factor (Refer 5.3.2 of IS:875(part 3-1987))

k3 = topographic factor (Refer 5.3.3 of IS:875(part 3-1987))

Wind coefficients

Wind speed $=21 \mathrm{~m} / \mathrm{s}$

Terrain category $=4$

Structure class $=\mathrm{B}$

Risk coefficient $(\mathrm{k} 1) \quad=1.0$

Topography $(\mathrm{k} 3)=1$

Wind pressure

Wind pressure on buildings is based on IS 875 Part-3 along with Finnish National Annex. In order to calculate the wind pressure on the building, several steps that are mentioned in this report are to be followed.

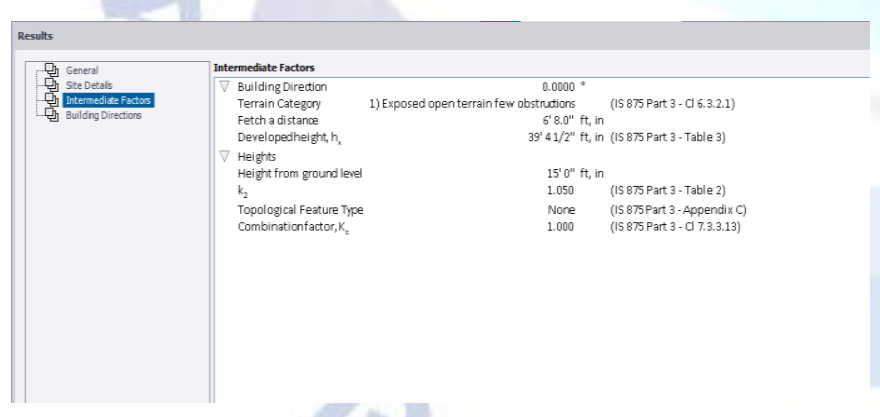

Figure 8: Intermediate Factors shows in software

\section{Basic wind velocity}

The basic wind velocity is calculated from the following equation:

Where:

$v$,is the fundamental value of the basic wind velocity $(\mathrm{m} / \mathrm{s})$

$\mathrm{C}$ is the direction factor, recommended value 1.0

$\mathrm{C}$ is the season factor, recommended value 1.0

It is assumed that the basic wind velocity

(obtained from meteorological data) is $21 \mathrm{~m} / \mathrm{s}$ $\mathrm{z}$,is the roughness height $\mathrm{z}$ at terrain category II

Terrain category III is considered for the project, so according to Table 4 below,

\begin{tabular}{|c|c|c|}
\hline \multicolumn{1}{|c|}{ Terrain category } & $\begin{array}{c}\mathbf{z}_{0} \\
\mathrm{~m}\end{array}$ & $\begin{array}{c}\mathbf{z}_{\min } \\
\mathrm{m}\end{array}$ \\
\hline $0 \quad$ Sea or coastal area exposed to the open sea & 0,003 & 1 \\
\hline I $\quad \begin{array}{l}\text { Lakes or flat and horizontal area with negligible vegetation and } \\
\text { without obstacles }\end{array}$ & 0,01 & 1 \\
\hline II $\quad \begin{array}{l}\text { Area with low vegetation such as grass and isolated obstacles } \\
\text { (trees, buildings) with separations of at least 20 obstacle heights }\end{array}$ & 0,05 & 2 \\
\hline III $\quad \begin{array}{l}\text { Area with regular cover of vegetation or buildings or with isolated } \\
\text { obstacles with separations of maximum 20 obstacle heights (such } \\
\text { as villages, suburban terrain, permanent forest) }\end{array}$ & 0,3 & 5 \\
\hline IV $\quad \begin{array}{l}\text { Area in which at least 15 \% of the surface is covered with buildings } \\
\text { and their average height exceeds 15 m }\end{array}$ & 1,0 & 10 \\
\hline NOTE: The terrain categories are illustrated in A.1. & \\
\hline
\end{tabular}

Table 4. Terrain categories and terrain parameters

\section{Seismic loading of the structure:}

Now the present effort the building is located in kadapa which come from under zone- III, by means of the IS:1893-1984, the following remain the various values for the building considered.

\section{Zone factor (Z):}

This one remains a factor towards obtain the design spectrum depending proceeding The basic zone factors included now this standard remain reasonable assessment of effective peak ground acceleration.

Zone factor $=0.16$ (Zone- III)

(From IS: 1893-2002 part-1, Table-2)

\section{Response reduction factor:}

This one is the factor by means of which the actual base shear force that would be generated if the structure were to remain elastic during its response to the Design Basics Earthquake (DBE) shaking, shall by reduced to obtain the design lateral force

Response reduction factor $=5.0$

(from IS: 1893-2002 part-1, Table-7.1)

\section{Importance factor:}

This one is a factor used toward obtain the design seismic force depending proceeding the functional use of the structure, characterized by hazardous consequences of post-earthquake practical necessity, ancient value, otherwise economic importance

Importance factor $=1.0$

(From IS: 1893-2002 part-1, Table-6

Snow load on the structure: 
In India, the load is to be considered in the Himalaya region where snow fall occurs. This is calculated as per IS 875 (Part 4): 1987. The snow load on the roof to be considered varies depending on the following points:

- The location (and orientation) of the building

- The slope of the roof

- The horizontal wind pressure on the roof

$$
s=\mu C C s
$$

Where:

$\mu \quad$ is the snow load shape coefficient

$\mathrm{C}$ is the exposure coefficient

$\mathrm{C}$ is the thermal coefficient

$\mathrm{s}$ is the characteristic value of snow load on the ground $(\mathrm{kN} / \mathrm{m})$

Table 5 shows the conditions based on the angle of the roof to determine the snow load shape coefficients.

Finnish National Annex recommends $\mathrm{C}=$ 1 as a value for the thermal coefficient.

\begin{tabular}{|c|c|c|c|}
\hline Angle of pitch of roof $\alpha$ & $0^{\circ} \leq \alpha \leq 30^{\circ}$ & $30^{\circ}<\alpha<60^{\circ}$ & $\alpha \geq 60^{\circ}$ \\
\hline$\mu_{1}$ & 0,8 & $0,8(60-\alpha) / 30$ & 0,0 \\
\hline$\mu_{2}$ & $0,8+0,8 \alpha / 30$ & 1,6 & -- \\
\hline
\end{tabular}

Table 5. Snow load shape coefficients

The exposure coefficient $\mathrm{C}$ can be obtained from Table 6 below. The location of the building is considered as "Normal topography". Table 6 . Recommended values of $\mathrm{Ce}$ for different topographies.

\begin{tabular}{|c|c|}
\hline Topography & $\bar{C}$ \\
\hline Windswept $^{a}$ & 0,8 \\
\hline Normal $^{b}$ & $(1,0)$ \\
\hline Sheltered $^{c}$ & 1,2 \\
\hline \multicolumn{2}{|c|}{$\begin{array}{l}\text { Windswept topography. flat unobstructed areas exposed on all sides } \\
\text { without, or little shelter afforded by terrain, higher construction works or } \\
\text { trees. }\end{array}$} \\
\hline \multicolumn{2}{|c|}{$\begin{array}{l}\text { Normal topography. areas where there is no significant removal of snow } \\
\text { by wind on construction work, because of terrain, other construction works } \\
\text { or trees. }\end{array}$} \\
\hline \multicolumn{2}{|c|}{$\begin{array}{l}\text { 'Sheltered topography. areas in which the construction work being } \\
\text { considered is considerably lower than the surrounding terrain or } \\
\text { surrounded by high trees and/or surrounded by higher construction works. }\end{array}$} \\
\hline
\end{tabular}

Table 6; Exposure coefficient C

\section{ANALYSIS RESULTS AND DISCUSSION}

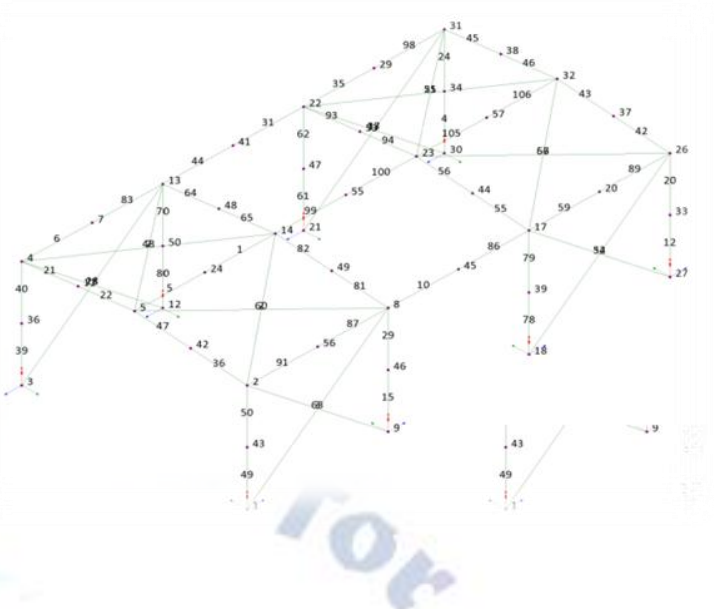

Figure 9.Shows Nodes in Tekla software

\section{Elements}

\begin{tabular}{|c|c|c|c|c|c|c|c|c|c|c|c|c|c|}
\hline t & $\begin{array}{r}\text { St } \\
\operatorname{ar} \\
t \\
N \\
\end{array}$ & $\begin{array}{l}\mathbf{E} \\
\mathbf{n} \\
\mathbf{d} \\
\mathbf{N}\end{array}$ & $\begin{array}{l}\text { Se } \\
\text { cti } \\
\text { on }\end{array}$ & $\begin{array}{c}\mathbf{G r} \\
\mathbf{a d} \\
\mathbf{e}\end{array}$ & $\begin{array}{c}\text { Const } \\
\text { ructio } \\
\text { n }\end{array}$ & $\begin{array}{c}\mathbf{F} \mathbf{1} \\
\mathbf{x} \\
\mathbf{D} \\
\mathbf{0}\end{array}$ & $\begin{array}{l}\mathbf{M} \\
\mathbf{1}_{\mathbf{x}} \\
\mathbf{D} \\
\mathbf{O}\end{array}$ & $\begin{array}{l}\mathbf{M} \\
\mathbf{1}_{\mathbf{y}} \\
\mathbf{D} \\
\mathbf{O}\end{array}$ & $\begin{array}{c}\mathbf{M} \\
\mathbf{1}_{\mathbf{z}} \\
\mathbf{D} \\
\mathbf{O}\end{array}$ & $\begin{array}{l}\mathbf{D} \\
\mathbf{O}\end{array}$ & $\begin{array}{l}\mathbf{M} \\
\mathbf{2}_{\mathbf{x}} \\
\mathbf{D} \\
\mathbf{O}\end{array}$ & $\begin{array}{l}\mathbf{y} \\
\mathbf{D} \\
\mathbf{O}\end{array}$ & \\
\hline 1 & $\begin{array}{l}2 \\
4\end{array}$ & 1 & $\begin{array}{l}\mathrm{B} \\
0\end{array}$ & \begin{tabular}{|l|}
$\mathrm{Fe}$ \\
41
\end{tabular} & & $\begin{array}{l}\mathrm{Fi} \\
\mathrm{xe}\end{array}$ & $\begin{array}{l}\mathrm{Fi} \\
\mathrm{xe}\end{array}$ & $\begin{array}{c}\mathrm{Fi} \\
\mathrm{xe}\end{array}$ & $\begin{array}{c}\mathrm{Fi} \\
\mathrm{xe}\end{array}$ & $\mathrm{Fi}$ & $\begin{array}{l}\mathrm{Fi} \\
\mathrm{xe}\end{array}$ & ee & \\
\hline 2 & 2 & $\begin{array}{l}1 \\
4\end{array}$ & $\begin{array}{l}10 \\
\mathrm{OL}\end{array}$ & $\begin{array}{l}\mathrm{Fe} \\
41\end{array}$ & & $\begin{array}{c}\mathrm{Fi} \\
\mathrm{xe}\end{array}$ & $\begin{array}{l}\mathrm{Fr} \\
\text { ee }\end{array}$ & & $\begin{array}{l}\mathrm{Fr} \\
\mathrm{ee}\end{array}$ & $\mathrm{Fi}$ & $\begin{array}{l}\mathrm{Fi} \\
\mathrm{xe}\end{array}$ & $\begin{array}{l}\mathrm{Fr} \\
\text { ee }\end{array}$ & \\
\hline 3 & 9 & 2 & $\begin{array}{l}10 \\
\mathrm{OL}\end{array}$ & $\begin{array}{l}\mathrm{Fe} \\
41\end{array}$ & & $\begin{array}{l}\mathrm{Fi} \\
\mathrm{xe}\end{array}$ & & $\begin{array}{l}\mathrm{Fr} \\
\text { ee }\end{array}$ & $\begin{array}{l}\text { Fr } \\
\text { ee }\end{array}$ & $\begin{array}{l}\mathrm{Fi} \\
\mathrm{xe}\end{array}$ & $\begin{array}{l}\mathrm{Fi} \\
\mathrm{xe}\end{array}$ & $\begin{array}{l}\mathrm{Fr} \\
\text { ee }\end{array}$ & \\
\hline 4 & $\begin{array}{l}3 \\
0\end{array}$ & 3 & $\mathrm{SC}$ & $\begin{array}{l}\mathrm{Fe} \\
41\end{array}$ & & $\begin{array}{l}\mathrm{Fi} \\
\mathrm{xe}\end{array}$ & $\mathrm{Fi}$ & $\begin{array}{c}\mathrm{Fi} \\
\mathrm{xe}\end{array}$ & $\mathrm{Fi}$ & $\begin{array}{l}\mathrm{Fi} \\
\mathrm{xe}\end{array}$ & $\begin{array}{l}\mathrm{Fi} \\
\mathrm{xe}\end{array}$ & $\begin{array}{c}\mathrm{Fi} \\
\mathrm{xe}\end{array}$ & \\
\hline 5 & 5 & & $\begin{array}{c}\mathrm{MB} \\
30\end{array}$ & $\begin{array}{l}\mathrm{Fe} \\
41\end{array}$ & & $\begin{array}{c}\mathrm{Fi} \\
\mathrm{xe}\end{array}$ & $\begin{array}{l}\mathrm{Fi} \\
\mathrm{xe}\end{array}$ & $\mathrm{Fr}$ & $\begin{array}{l}\text { Fr } \\
\text { ee }\end{array}$ & $\begin{array}{l}\mathrm{Fi} \\
\mathrm{xe}\end{array}$ & $\begin{array}{l}\text { Fi } \\
x \mathrm{xe}\end{array}$ & $\begin{array}{c}\mathrm{Fi} \\
\mathrm{xe}\end{array}$ & \\
\hline 6 & 4 & 7 & $\begin{array}{l}\mathrm{B} \\
0\end{array}$ & & & $\begin{array}{l}\mathrm{Fi} \\
\mathrm{xe}\end{array}$ & & & & $\begin{array}{l}\mathrm{Fi} \\
\mathrm{xe}\end{array}$ & $\begin{array}{l}\mathrm{Fi} \\
\mathrm{xe}\end{array}$ & $\begin{array}{c}\mathrm{Fi} \\
\mathrm{xe}\end{array}$ & \\
\hline 7 & $\begin{array}{l}1 \\
4\end{array}$ & 4 & 0 & & & & $\begin{array}{l}\mathrm{Fr} \\
\mathrm{ee}\end{array}$ & & & $\begin{array}{l}F \\
x \epsilon\end{array}$ & $\begin{array}{l}\mathrm{Fi} \\
\mathrm{xe}\end{array}$ & $\begin{array}{l}\mathrm{Fr} \\
\text { ee }\end{array}$ & \\
\hline 10 & 8 & & $\mathrm{MB}$ & $\begin{array}{l}\mathrm{Fe} \\
41\end{array}$ & & $\begin{array}{c}\mathrm{Fi} \\
\mathrm{xe}\end{array}$ & $\mathrm{xe}$ & ee & ee & $\begin{array}{l}\text { Fi } \\
\text { xe }\end{array}$ & $\begin{array}{l}\mathrm{Fi} \\
\mathrm{xe}\end{array}$ & $\begin{array}{c}\mathrm{Fi} \\
\mathrm{xe}\end{array}$ & \\
\hline 11 & $\begin{array}{l}1 \\
2\end{array}$ & 4 & $\mathrm{~L}$ & & & $\begin{array}{l}\mathrm{Fi} \\
\mathrm{xe}\end{array}$ & & & $\mathrm{Fr}$ & $\mathrm{xe}$ & $\begin{array}{l}\mathrm{Fi} \\
\mathrm{xe}\end{array}$ & $\begin{array}{l}\mathrm{Fr} \\
\mathrm{ee}\end{array}$ & \\
\hline 12 & $\begin{array}{l}2 \\
7\end{array}$ & $\begin{array}{l}3 \\
3\end{array}$ & 5 & $\begin{array}{l}\mathrm{Fe} \\
41\end{array}$ & & $\mathrm{Fi}$ & & & & F & $\begin{array}{l}\mathrm{Fi} \\
\mathrm{xe}\end{array}$ & $\mathrm{Fi}$ & \\
\hline 15 & 9 & 4 & $\begin{array}{c}\mathrm{HB} \\
45\end{array}$ & $\begin{array}{l}\mathrm{Fe} \\
41\end{array}$ & & $\begin{array}{l}\mathrm{Fi} \\
\mathrm{xe}\end{array}$ & & $\begin{array}{l}\mathrm{Fi} \\
\mathrm{xe}\end{array}$ & & xe & $\begin{array}{l}\mathrm{Fi} \\
\mathrm{xe}\end{array}$ & $\begin{array}{l}\mathrm{F} 1 \\
\mathrm{xe}\end{array}$ & \\
\hline 17 & $\begin{array}{l}2 \\
1\end{array}$ & 3 & $\begin{array}{l}10 \\
\text { OL }\end{array}$ & $\begin{array}{l}\mathrm{Fe} \\
41\end{array}$ & & $\mathrm{Fi}$ & & & $\mathrm{Fr}$ & $\mathrm{Fi}$ & $\mathrm{Fi}$ & $\mathrm{Fr}$ & \\
\hline
\end{tabular}




\begin{tabular}{|c|c|c|c|c|c|c|c|c|c|c|c|c|c|}
\hline $\begin{array}{c}\text { Ele } \\
\text { me } \\
\text { nt } \\
\mathrm{Nu}\end{array}$ & $\begin{array}{c}\text { St } \\
\text { ar } \\
\mathbf{t} \\
\mathbf{N}\end{array}$ & $\begin{array}{l}\mathbf{E} \\
\mathbf{n} \\
\mathbf{d} \\
\mathbf{N}\end{array}$ & $\begin{array}{l}\text { Se } \\
\text { cti } \\
\text { on }\end{array}$ & $\begin{array}{c}\mathbf{G r} \\
\mathbf{a d} \\
\mathbf{e}\end{array}$ & $\begin{array}{c}\text { Const } \\
\text { ructio } \\
n\end{array}$ & $\begin{array}{c}F^{1} \\
\mathbf{x} \\
\mathbf{D} \\
\mathbf{O}\end{array}$ & $\begin{array}{l}\mathbf{M} \\
1_{\mathbf{x}} \\
\mathbf{D} \\
\mathbf{O}\end{array}$ & $\begin{array}{c}\mathbf{M} \\
1_{\mathbf{y}} \\
\mathbf{D} \\
\mathbf{O}\end{array}$ & $\begin{array}{c}\mathbf{M} \\
\mathbf{1}_{\mathbf{z}} \\
\mathbf{D} \\
\mathbf{O}\end{array}$ & $\begin{array}{c}\mathbf{F}^{2} \\
\mathbf{x} \\
\mathbf{D} \\
\mathbf{O}\end{array}$ & $\begin{array}{l}\mathbf{M} \\
\mathbf{2}_{\mathbf{x}} \\
\mathbf{D} \\
\mathbf{O}\end{array}$ & $\begin{array}{l}\mathbf{M} \\
\mathbf{2}_{\mathbf{y}} \\
\mathbf{D} \\
\mathbf{O}\end{array}$ & $\begin{array}{l}\mathbf{M} \\
\mathbf{2}_{z} \\
\mathbf{D} \\
\mathbf{O}\end{array}$ \\
\hline \multirow[b]{2}{*}{20} & 3 & 2 & SC & $\mathrm{Fe}$ & on-c & $\mathrm{Fi}$ & $\mathrm{Fi}$ & $\mathrm{Fi}$ & $\mathrm{Fi}$ & $\mathrm{Fi}$ & $\mathrm{Fi}$ & $\mathrm{Fi}$ & $\mathrm{Fi}$ \\
\hline & 3 & 6 & 25 & 41 & npos & & xe & $\mathrm{xe}$ & $\mathrm{xe}$ & $\mathrm{xe}$ & $\mathrm{xe}$ & $\mathrm{xe}$ & $\mathrm{xe}$ \\
\hline \multirow[b]{2}{*}{21} & & 1 & MB & $\mathrm{Fe}$ & Non-c & $\mathrm{Fi}$ & $\mathrm{Fi}$ & $\mathrm{Fi}$ & $\mathrm{Fi}$ & $\mathrm{Fi}$ & $\mathrm{Fi}$ & $\mathrm{Fi}$ & $\mathrm{Fi}$ \\
\hline & 4 & 1 & 40 & 41 & ompos & & $\mathrm{xe}$ & $\mathrm{xe}$ & $\mathrm{xe}$ & $\mathrm{xe}$ & $\mathrm{xe}$ & $\mathrm{xe}$ & $x e$ \\
\hline \multirow[b]{2}{*}{22} & & & MB & $\mathrm{Fe}$ & on-c & $\mathrm{Fi}$ & $\mathrm{Fi}$ & $\mathrm{Fi}$ & $\mathrm{Fi}$ & $\mathrm{Fi}$ & $\mathrm{Fi}$ & $\mathrm{Fi}$ & $\mathrm{Fi}$ \\
\hline & & 5 & 40 & 41 & ompos & & xe & $\mathrm{xe}$ & $\mathrm{xe}$ & $\mathrm{xe}$ & $\mathrm{xe}$ & $\mathrm{xe}$ & $x e$ \\
\hline \multirow{3}{*}{24} & & 3 & SC & $\mathrm{Fe}$ & Non-c & $\mathrm{Fi}$ & $\mathrm{Fi}$ & $\mathrm{Fi}$ & $\mathrm{Fi}$ & $\frac{\mathrm{d}}{\mathrm{Fi}}$ & Fi & $\begin{array}{l}\mathrm{d} \\
\mathrm{Fi}\end{array}$ & $\mathrm{Fi}$ \\
\hline & & 3 & 25 & 41 & ompos & $\mathrm{xe}$ & $\mathrm{xe}$ & $\mathrm{xe}$ & xe & xe & xe & $\mathrm{xe}$ & $\mathrm{xe}$ \\
\hline & & & & & & & d & d & له & d. & A. & لـ & له \\
\hline \multirow{2}{*}{25} & 2 & 3 & 10 & $\mathrm{Fe}$ & Steel & $\mathrm{Fi}$ & $\mathrm{Fr}$ & $\mathrm{Fr}$ & $\mathrm{Fr}$ & $\mathrm{Fi}$ & $\mathrm{Fi}$ & $\mathrm{Fr}$ & $\mathrm{Fr}$ \\
\hline & 3 & 1 & OL & 41 & brace & $\mathrm{xe}$ & ee & ee & ee & xe & xe & ee & ee \\
\hline \multirow{2}{*}{28} & & 1 & 10 & $\mathrm{Fe}$ & Steel & $\mathrm{Fi}$ & $\mathrm{Fr}$ & $\mathrm{Fr}$ & $\mathrm{Fr}$ & $\mathrm{Fi}$ & $\mathrm{Fi}$ & $\mathrm{Fr}$ & $\mathrm{Fr}$ \\
\hline & 3 & 3 & $\mathrm{OL}$ & 41 & brace & $\mathrm{xe}$ & ee & ee & ee & $\mathrm{xe}$ & $\mathrm{xe}$ & ee & ee \\
\hline \multirow{2}{*}{29} & & & $\mathrm{HB}$ & $\mathrm{Fe}$ & on-c & $\mathrm{Fi}$ & $\mathrm{Fi}$ & $\mathrm{Fi}$ & $\mathrm{Fi}$ & $\mathrm{Fi}$ & $\mathrm{Fi}$ & $\mathrm{Fi}$ & $\mathrm{Fi}$ \\
\hline & 6 & 8 & 45 & 41 & ompos & $\mathrm{xe}$ & $\mathrm{xe}$ & $\mathrm{xe}$ & $\mathrm{xe}$ & xe & $\mathrm{xe}$ & xe & $\mathrm{xe}$ \\
\hline \multirow{3}{*}{31} & & 2 & MB & $\mathrm{Fe}$ & Non-c & $\mathrm{Fi}$ & $\mathrm{Fi}$ & $\mathrm{Fi}$ & $\mathrm{Fi}$ & Fi & $\mathrm{Fi}$ & $\mathrm{Fr}$ & $\mathrm{Fr}$ \\
\hline & 1 & 2 & 30 & 41 & ompos & $\mathrm{xe}$ & $\mathrm{xe}$ & $\mathrm{xe}$ & $\mathrm{xe}$ & xe & $\mathrm{xe}$ & ee & ee \\
\hline & & & & $\mathrm{Fe}$ & & & $\Gamma_{0}$ & $d$ & $\Gamma$ & Fi & $\mathrm{Fi}$ & & \\
\hline \multirow[t]{2}{*}{32} & 1 & 2 & 10 & $\mid \begin{array}{l}1 \\
41\end{array}$ & el & 11 & $\mathrm{Fr}$ & $\mathrm{Fr}$ & $\mathrm{Fr}$ & 11 & 11 & $\mathrm{Fr}$ & $\mathrm{Fr}$ \\
\hline & & 6 & OL & 41 & & $\mathrm{Xe}$ & ee & ee & ee & de & $x \in$ & ee & ee \\
\hline \multirow{2}{*}{33} & 3 & 2 & 10 & $\mathrm{Fe}$ & Steel & $\mathrm{Fi}$ & $\mathrm{Fr}$ & $\mathrm{Fr}$ & $\mathrm{Fr}$ & $\mathrm{Fi}$ & $\mathrm{Fi}$ & $\mathrm{Fr}$ & $\mathrm{Fr}$ \\
\hline & 0 & 2 & $\mathrm{OL}$ & 41 & brace & $\mathrm{xe}$ & ee & ee & ee & xe & xe & ee & ee \\
\hline \multirow[b]{2}{*}{35} & 2 & 2 & MB & $\mathrm{Fe}$ & Non-c & $\mathrm{Fi}$ & $\mathrm{Fi}$ & $\mathrm{Fr}$ & $\mathrm{Fr}$ & Fi & $\mathrm{Fi}$ & $\mathrm{Fi}$ & $\mathrm{Fi}$ \\
\hline & 2 & 9 & 20 & 41 & ompos & $\mathrm{xe}$ & $\mathrm{xe}$ & ee & eel & $\mathrm{xe}$ & $\mathrm{xe}$ & $\mathrm{xe}$ & $\mathrm{xe}$ \\
\hline \multirow[b]{2}{*}{36} & & & MB & $\mathrm{Fe}$ & Non-c & $\mathrm{Fi}$ & Fi & $\mathrm{Fi}$ & $\mathrm{Fi}$ & \begin{tabular}{|l} 
\\
$\mathrm{Fi}$
\end{tabular} & $\frac{d}{\mathrm{Fi}}$ & $\mathrm{Fi}$ & $\mathrm{Fi} \mid$ \\
\hline & 2 & 2 & 40 & 41 & ompos & $\mathrm{xe}$ & $\mathrm{xe}$ & $\mathrm{xe}$ & $\mathrm{xe}$ & xe & $\mathrm{xe}$ & xe & $x e$ \\
\hline \multirow[b]{2}{*}{39} & & 3 & SC & $\mathrm{Fe}$ & Non-c & $\mathrm{Fi}$ & $\mathrm{Fi}$ & $\mathrm{Fi}$ & $\mathrm{Fi}$ & Fi & $\mathrm{Fi}$ & $\mathrm{Fi}$ & $\mathrm{Fi}$ \\
\hline & 3 & 6 & 25 & 41 & ompos & $\mathrm{xe}$ & $\mathrm{xe}$ & $\mathrm{xe}$ & xe & xe & $\mathrm{xe}$ & xe & $x e$ \\
\hline \multirow[b]{2}{*}{40} & & & SC & $\mathrm{Fe}$ & Non-c & $\mathrm{Fi}$ & $\mathrm{Fi}$ & $\mathrm{Fi}$ & $\mathrm{Fi}$ & $\mathrm{Fi}$ & $\mathrm{Fi}$ & $\mathrm{Fi}$ & $\mathrm{Fi}$ \\
\hline & & 4 & 25 & 41 & $\mathrm{bs}$ & $\mathrm{xe}$ & xel & $\mathrm{xe}$ & xel & xe & $\mathrm{xe}$ & $\mathrm{xe}$ & $\mathrm{xe}$ \\
\hline \multirow{2}{*}{42} & 2 & 3 & $\mathrm{MB}$ & $\mathrm{Fe}$ & Non-c & $\mathrm{Fi}$ & $\mathrm{Fi}$ & $\mathrm{Fi}$ & $\mathrm{Fi}$ & Fi & $\mathrm{Fi}$ & $\mathrm{Fi}$ & $\mathrm{Fi}$ \\
\hline & & 7 & 40 & 41 & os & $\mathrm{xe}$ & $\mathrm{xe}$ & $\mathrm{xe}$ & $\mathrm{xe}$ & xe & $\mathrm{xe}$ & xe & $\mathrm{xe}$ \\
\hline \multirow{3}{*}{43} & & 2 & $\mathrm{MB}$ & $\mathrm{Fe}$ & & $\mathrm{F}$ & $\mathrm{Fi}$ & $\mathrm{Fi}$ & Fi & \begin{tabular}{|l}
$\mathrm{Fi}$ \\
$\mathrm{Fi}$
\end{tabular} & $\mathrm{Fi}$ & $\mathrm{F}$ & $\mathrm{H}$ \\
\hline & 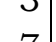 & 3 & 40 & $1 \mid$ & & & $\mathrm{xe}$ & $\mathrm{xe}$ & $\mathrm{xe}$ & $x e$ & $\mathrm{xe}$ & & $\mathrm{X} \epsilon$ \\
\hline & & 2 & & & & & & & $\mathrm{xe}^{2}$ & & $x \in$ & & $x$ \\
\hline \multirow{2}{*}{44} & 1 & 4 & MB & $\mathrm{Fe}$ & Non-c & $\mathrm{Fi}$ & $\mathrm{Fi}$ & $\mathrm{Fr}$ & $\mathrm{Fr}$ & $\mathrm{Fi}$ & $\mathrm{Fi}$ & $\mathrm{Fi}$ & $\mathrm{Fi}$ \\
\hline & & & 30 & 41 & ompos & $\mathrm{xe}$ & $\mathrm{xe}$ & ee & eel & xe & $\mathrm{xe}$ & $x \epsilon$ & $x e$ \\
\hline \multirow[b]{2}{*}{45} & 3 & 3 & MB & $\mathrm{Fe}$ & Non-c & $\mathrm{Fi}$ & $\mathrm{Fi}$ & $\mathrm{Fi}$ & $\mathrm{Fi}$ & $\mathrm{Fi}$ & Fi & $\mathrm{Fi}$ & $\mathrm{Fi}$ \\
\hline & & 8 & 40 & 41 & ompos & $\mathrm{xe}$ & xe & $\mathrm{xe}$ & $\mathrm{xe}$ & $\mathrm{xe}$ & $\mathrm{xe}$ & xe & $\mathrm{xe}$ \\
\hline \multirow{3}{*}{46} & & & MB & $\mathrm{Fe}$ & Non-c & $\mathrm{Fi}$ & $\mathrm{Fi}$ & $\mathrm{Fi}$ & $\mathrm{Fi}$ & $\mathrm{Fi}$ & $\mathrm{Fi}$ & $\mathrm{Fi}$ & $\mathrm{Fi}$ \\
\hline & & ? & 40 & 41 & & & $\mathrm{xe}$ & xel & 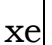 & $\mathrm{xe}$ & $\mathrm{xe}$ & xe| & $x \in$ \\
\hline & & & & & & & & & & & & & \\
\hline \multirow[b]{2}{*}{47} & & & MB & $\mathrm{Fe}$ & Non-c & $\mathrm{Fi}$ & $\mathrm{Fi}$ & $\mathrm{Fi}$ & $\mathrm{Fi}$ & $\mathrm{Fi}$ & $\mathrm{Fi}$ & $\mathrm{Fi}$ & $\mathrm{Fi}$ \\
\hline & & & 40 & 41 & os & & & & & & & & \\
\hline
\end{tabular}

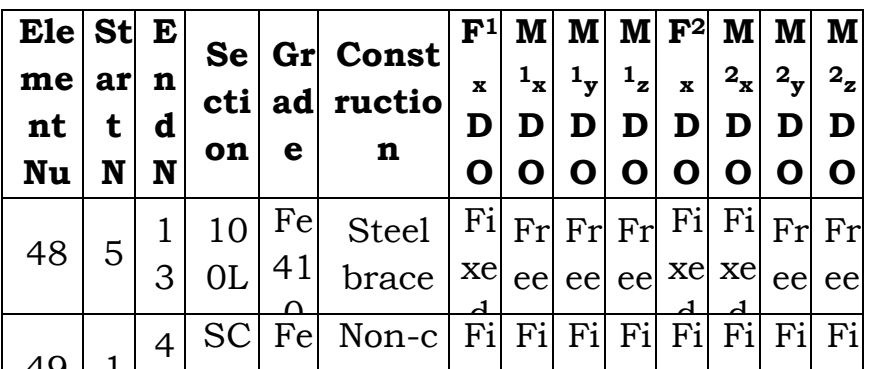

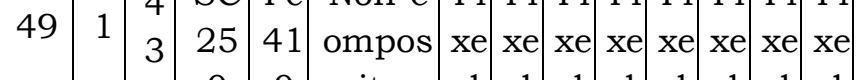

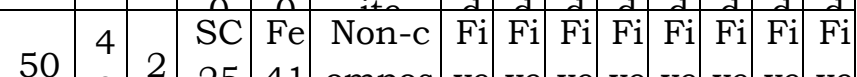

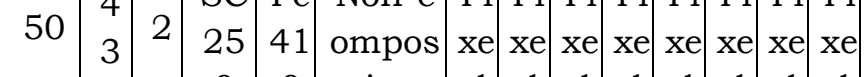

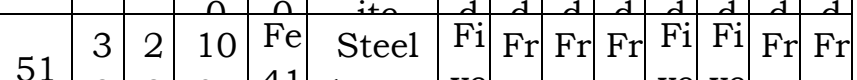

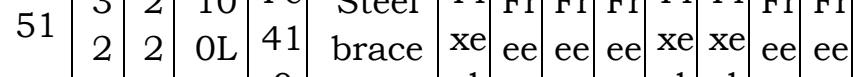

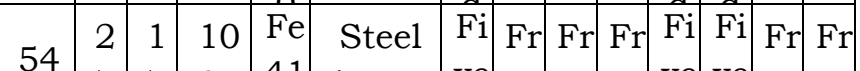

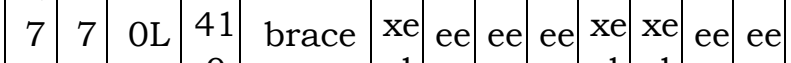

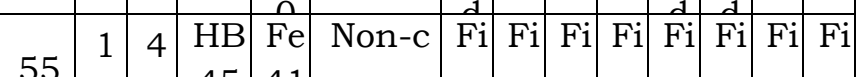

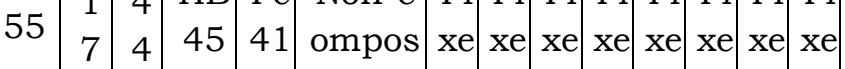

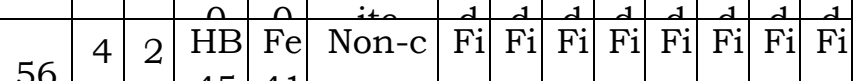

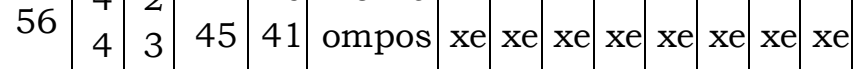

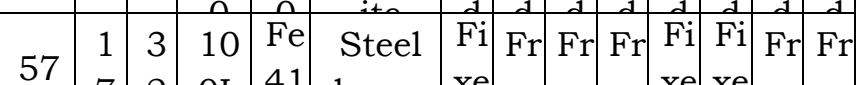

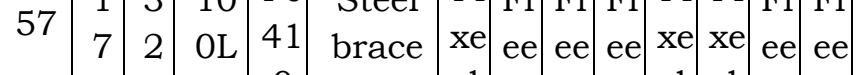

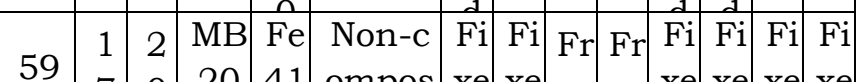

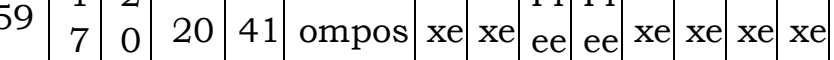

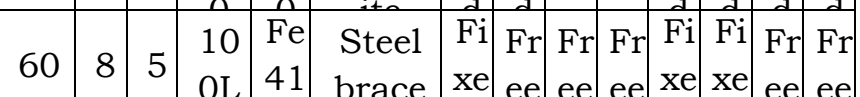

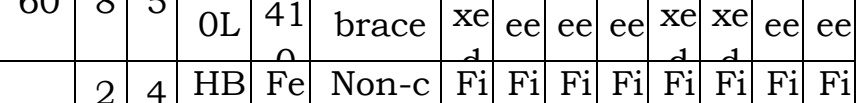

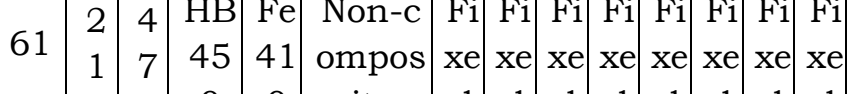

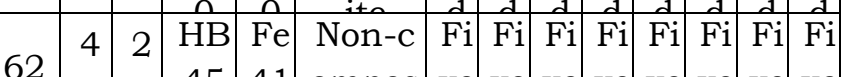

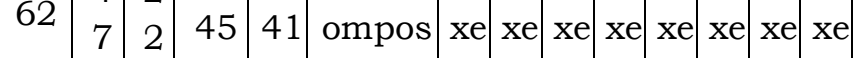

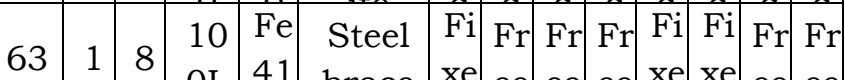

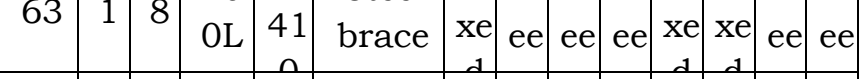

$\begin{array}{lllllllllllllll}64 & 1 & 4 & \text { HB } & \text { Fe } & \text { Non-C } & \text { Fi } & \text { Fi } & \text { Fi } & \text { Fi } & \text { Fi } & \text { Fi } & \text { Fi } & \text { Fi }\end{array}$

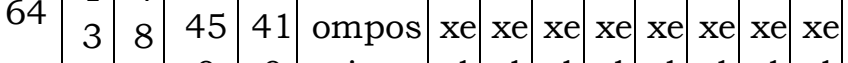

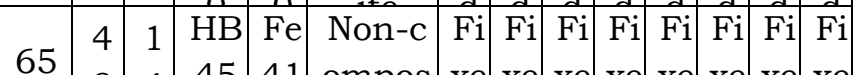

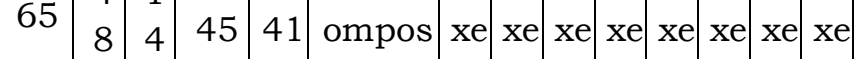

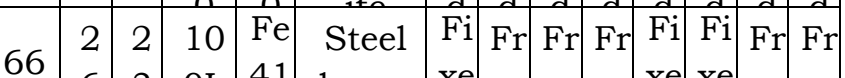

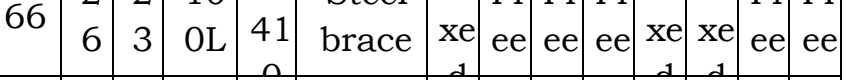

\begin{tabular}{lll|l|lllllllllll}
70 & 5 & 1 & HB & Fe & Non-c & Fi & Fi & Fi & Fi & Fi & Fi & Fi & Fi
\end{tabular}

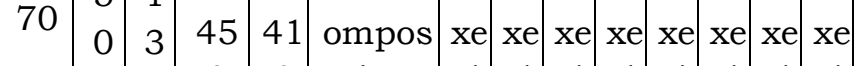

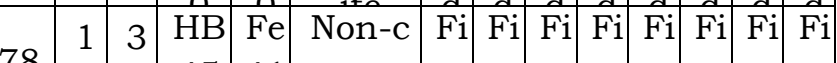

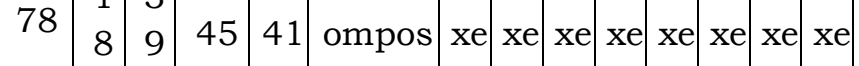

\begin{tabular}{llllllllllllll}
79 & 3 & 1 & $\mathrm{HB}$ & $\mathrm{Fe}$ & Non-c & Fi & Fi & Fi & Fi & Fi & d & A & $d$ \\
\hline
\end{tabular}

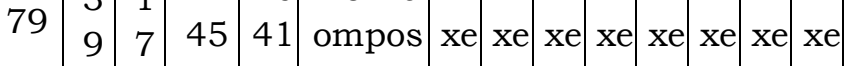

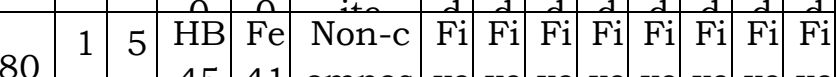

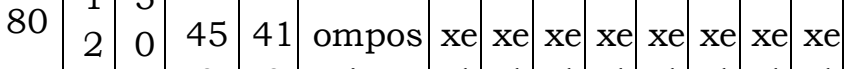




\begin{tabular}{|c|c|c|c|c|c|c|c|c|c|c|c|c|c|}
\hline $\begin{array}{c}\text { Ele } \\
\text { me } \\
\text { nt } \\
\text { Nu }\end{array}$ & $\begin{array}{c}\mathbf{S t} \\
\mathbf{a r} \\
\mathbf{t} \\
\mathrm{N}\end{array}$ & $\begin{array}{l}\mathbf{E} \\
\mathbf{n} \\
\mathbf{d} \\
\mathbf{N}\end{array}$ & $\begin{array}{l}\text { Se } \\
\text { cti } \\
\text { on }\end{array}$ & $\begin{array}{c}\mathbf{G r} \\
\mathbf{a d} \\
\mathbf{e}\end{array}$ & $\begin{array}{c}\text { Const } \\
\text { ructio } \\
\text { n }\end{array}$ & $\begin{array}{c}\mathbf{F}^{\mathbf{1}} \\
\mathbf{x} \\
\mathbf{D} \\
\mathbf{O}\end{array}$ & $\begin{array}{c}\mathbf{M} \\
\mathbf{1}_{\mathbf{x}} \\
\mathbf{D} \\
\mathbf{O}\end{array}$ & $\begin{array}{l}\mathbf{M} \\
\mathbf{1}_{\mathbf{y}} \\
\mathbf{D} \\
\mathbf{O}\end{array}$ & \begin{tabular}{l|}
$\mathbf{M}$ \\
$\mathbf{1}_{\mathbf{z}}$ \\
$\mathbf{D}$ \\
$\mathbf{O}$
\end{tabular} & \begin{tabular}{r|r}
$\mathbf{F}^{2}$ & $\mathbf{1}$ \\
$\mathbf{x}$ & \\
$\mathbf{D}$ & \\
$\mathbf{O}$ & \\
\end{tabular} & $\begin{array}{l}\mathbf{M} \\
\mathbf{2}_{\mathbf{x}} \\
\mathbf{D} \\
\mathbf{O}\end{array}$ & $\begin{array}{l}\mathbf{M} \\
\mathbf{2}_{\mathrm{y}} \\
\mathbf{D} \\
\mathbf{O}\end{array}$ & $\begin{array}{l}\text { M } \\
2_{z} \\
\mathrm{D} \\
\mathrm{O}\end{array}$ \\
\hline 81 & 8 & $\begin{array}{l}4 \\
9\end{array}$ & \begin{tabular}{|c|}
$\mathrm{HB}$ \\
45
\end{tabular} & $\begin{array}{l}\mathrm{Fe} \\
41\end{array}$ & $\begin{array}{l}\text { Non-c } \\
\text { ompos }\end{array}$ & $\begin{array}{l}\mathrm{Fi} \\
\mathrm{xe}\end{array}$ & $\begin{array}{l}\mathrm{Fi} \\
\mathrm{xe}\end{array}$ & $\begin{array}{c}\mathrm{Fi} \\
\mathrm{xe}\end{array}$ & $\begin{array}{c}\mathrm{Fi} \\
\mathrm{xe}\end{array}$ & \begin{tabular}{l|l}
$\mathrm{Fi}$ & 1 \\
$\mathrm{xe}$ & $\mathrm{x}$
\end{tabular} & $\begin{array}{c}\mathrm{Fi} \\
\mathrm{xe}\end{array}$ & $\begin{array}{l}\mathrm{Fi} \\
\mathrm{xe}\end{array}$ & $\begin{array}{l}\mathrm{Fi} \\
\mathrm{xe}\end{array}$ \\
\hline 82 & & $\begin{array}{l}1 \\
4\end{array}$ & $\begin{array}{r}\mathrm{HB} \\
45\end{array}$ & $\begin{array}{l}\mathrm{Fe} \\
41\end{array}$ & $\begin{array}{l}\text { Non-c } \\
\text { ompos }\end{array}$ & $\begin{array}{l}\mathrm{Fi} \\
\mathrm{xe}\end{array}$ & $\begin{array}{c}\mathrm{Fi} \\
\mathrm{xe}\end{array}$ & $\begin{array}{c}\mathrm{Fi} \\
\mathrm{xe}\end{array}$ & $\begin{array}{c}\mathrm{Fi} \\
\mathrm{xe}\end{array}$ & \begin{tabular}{c|c}
$\mathrm{Fi}$ & \\
$\mathrm{xe}$ & $\mathrm{x}$
\end{tabular} & $\begin{array}{c}\mathrm{Fi} \\
\mathrm{xe}\end{array}$ & $\begin{array}{c}\mathrm{Fi} \\
\mathrm{xe}\end{array}$ & $\begin{array}{l}\text { Fi } \\
\text { xe }\end{array}$ \\
\hline 83 & 7 & $\begin{array}{l}1 \\
3\end{array}$ & $\begin{array}{r}\mathrm{MB} \\
20\end{array}$ & $\begin{array}{l}\mathrm{Fe} \\
41\end{array}$ & $\begin{array}{l}\text { Non-c } \\
\text { ompos }\end{array}$ & $\begin{array}{c}\mathrm{Fi} \\
\mathrm{xe}\end{array}$ & $\begin{array}{c}\mathrm{Fi} \\
\mathrm{xe}\end{array}$ & $\begin{array}{c}\mathrm{Fi} \\
\mathrm{xe}\end{array}$ & $\begin{array}{l}F i \\
x e\end{array}$ & \begin{tabular}{c|c}
$\mathrm{Fi}$ & \\
$\mathrm{xe}$ & $\mathrm{x}$
\end{tabular} & $\begin{array}{c}\mathrm{Fi} \\
\mathrm{xe}\end{array}$ & $\begin{array}{l}\mathrm{Fr} \\
\text { ee }\end{array}$ & $\begin{array}{l}\mathrm{Fr} \\
\text { ee }\end{array}$ \\
\hline 86 & & $\begin{array}{l}1 \\
7\end{array}$ & $\begin{array}{c}\text { MB } \\
30\end{array}$ & $\begin{array}{l}\mathrm{Fe} \\
41\end{array}$ & $\begin{array}{l}\text { Non-c } \\
\text { ompos }\end{array}$ & $\begin{array}{l}\mathrm{Fi} \\
\mathrm{xe}\end{array}$ & $\begin{array}{c}\mathrm{Fi} \\
\mathrm{xe}\end{array}$ & $\begin{array}{c}\mathrm{Fi} \\
\mathrm{xe}\end{array}$ & $\begin{array}{l}\mathrm{Fi} \\
\mathrm{xe}\end{array}$ & $\begin{array}{l}\mathrm{Fi} \\
\mathrm{xe}\end{array}$ & $\begin{array}{l}\mathrm{Fi} \\
\mathrm{xe}\end{array}$ & $\begin{array}{l}\mathrm{Fr} \\
\mathrm{ee}\end{array}$ & $\begin{array}{l}\mathrm{Fr} \\
\mathrm{ee}\end{array}$ \\
\hline 87 & $\begin{array}{l}5 \\
6\end{array}$ & 8 & $\begin{array}{r}\mathrm{MB} \\
20\end{array}$ & $\begin{array}{l}\mathrm{Fe} \\
41\end{array}$ & $\begin{array}{l}\text { Non-c } \\
\text { ompos }\end{array}$ & $\begin{array}{l}\mathrm{Fi} \\
\mathrm{xe}\end{array}$ & $\begin{array}{l}\mathrm{Fi} \\
\mathrm{xe}\end{array}$ & $\begin{array}{c}\mathrm{Fi} \\
\mathrm{xe}\end{array}$ & $\begin{array}{l}\mathrm{Fi} \\
\mathrm{xe}\end{array}$ & \begin{tabular}{c|}
$\mathrm{Fi}$ \\
$\mathrm{xe}$
\end{tabular} & $\begin{array}{l}\mathrm{Fi} \\
\mathrm{xe}\end{array}$ & & $\begin{array}{l}\mathrm{Fr} \\
\text { ee }\end{array}$ \\
\hline 89 & $\begin{array}{l}2 \\
0\end{array}$ & $\begin{array}{l}2 \\
6\end{array}$ & $\begin{array}{c}\text { MB } \\
20\end{array}$ & $\begin{array}{l}\mathrm{Fe} \\
41\end{array}$ & $\begin{array}{l}\text { Non-c } \\
\text { ompos }\end{array}$ & & $\begin{array}{l}\mathrm{Fi} \\
\mathrm{xe}\end{array}$ & $\begin{array}{l}\mathrm{Fi} \\
\mathrm{xe}\end{array}$ & $\begin{array}{l}\mathrm{Fi} \\
\mathrm{xe}\end{array}$ & $\begin{array}{l}\text { Fi } \\
\text { xe }\end{array}$ & $\begin{array}{l}\mathrm{Fi} \\
\mathrm{xe}\end{array}$ & & \\
\hline 91 & 2 & $\begin{array}{l}5 \\
6\end{array}$ & & & $\begin{array}{l}\text { Non-c } \\
\text { ompos }\end{array}$ & & $\begin{array}{l}\mathrm{Fi} \\
\mathrm{xe}\end{array}$ & & $\mathrm{F}_{1}$ & $\begin{array}{l}\text { Fi } \\
\text { xe }\end{array}$ & $\begin{array}{l}\mathrm{Fi} \\
\mathrm{xe}\end{array}$ & & \\
\hline 93 & $\begin{array}{l}2 \\
2\end{array}$ & $\begin{array}{l}5 \\
3\end{array}$ & $\begin{array}{r}\mathrm{HB} \\
45\end{array}$ & $\begin{array}{l}\mathrm{Fe} \\
41\end{array}$ & $\begin{array}{l}\text { Non-c } \\
\text { ompos }\end{array}$ & $\begin{array}{l}\mathrm{Fi} \\
\mathrm{xe}\end{array}$ & $\begin{array}{l}\mathrm{Fi} \\
\mathrm{xe}\end{array}$ & & $\begin{array}{l}\mathrm{Fi} \\
\mathrm{xe}\end{array}$ & $\begin{array}{l}\mathrm{d} \\
\mathrm{Fi} \\
\mathrm{xe}\end{array}$ & $\begin{array}{l}\mathrm{Fi} \\
\mathrm{xe}\end{array}$ & & $\begin{array}{l}\mathrm{Fi} \\
\mathrm{xe}\end{array}$ \\
\hline 94 & $\begin{array}{l}5 \\
3\end{array}$ & $\begin{array}{l}2 \\
3\end{array}$ & $\begin{array}{r}\mathrm{HB} \\
45\end{array}$ & $\begin{array}{l}\mathrm{Fe} \\
41\end{array}$ & $\begin{array}{l}\text { Non-c } \\
\text { ompos }\end{array}$ & & $\begin{array}{l}\mathrm{Fi} \\
\mathrm{xe}\end{array}$ & & $\begin{array}{l}\mathrm{Fi} \\
\mathrm{xe}\end{array}$ & \begin{tabular}{c|}
$\mathrm{Fi}$ \\
$\mathrm{xe}$
\end{tabular} & $\begin{array}{l}\mathrm{Fi} \\
\mathrm{xe}\end{array}$ & $\begin{array}{c}\mathrm{Fi} \\
\mathrm{xe}\end{array}$ & $\begin{array}{l}\mathrm{Fi} \\
\mathrm{xe}\end{array}$ \\
\hline 98 & $\begin{array}{l}2 \\
9\end{array}$ & $\begin{array}{l}3 \\
1\end{array}$ & $\begin{array}{r}\mathrm{MB} \\
20\end{array}$ & $\begin{array}{l}\mathrm{Fe} \\
41\end{array}$ & $\begin{array}{l}\text { Non-c } \\
\text { ompos }\end{array}$ & $\begin{array}{l}\mathrm{Fi} \\
\mathrm{xe}\end{array}$ & $\begin{array}{l}\mathrm{Fi} \\
\mathrm{xe}\end{array}$ & $\begin{array}{c}\mathrm{Fi} \\
\mathrm{xe}\end{array}$ & $\begin{array}{l}\mathrm{Fi} \\
\mathrm{xe}\end{array}$ & $\begin{array}{l}\text { Fi } \\
x e\end{array}$ & $\begin{array}{l}\mathrm{Fi} \\
\mathrm{xe}\end{array}$ & $\begin{array}{l}\mathrm{Fr} \\
\text { ee }\end{array}$ & $\begin{array}{l}\mathrm{Fr} \\
\text { ee }\end{array}$ \\
\hline 99 & $\begin{array}{l}1 \\
4\end{array}$ & $\begin{array}{l}5 \\
5\end{array}$ & $\begin{array}{r}\mathrm{MB} \\
30\end{array}$ & $\begin{array}{l}\mathrm{Fe} \\
41\end{array}$ & $\begin{array}{l}\text { Non-c } \\
\text { ompos }\end{array}$ & $\begin{array}{l}\mathrm{Fi} \\
\mathrm{xe}\end{array}$ & $\begin{array}{l}\mathrm{Fi} \\
\mathrm{xe}\end{array}$ & $\begin{array}{l}\mathrm{Fr} \\
\text { ee }\end{array}$ & $\begin{array}{l}\mathrm{Fr} \\
\text { ee }\end{array}$ & $\begin{array}{l}\mathrm{Fi} \\
\mathrm{xe}\end{array}$ & $\begin{array}{l}\mathrm{Fi} \\
\mathrm{xe}\end{array}$ & $\begin{array}{l}\mathrm{Fi} \\
\mathrm{xe}\end{array}$ & $\begin{array}{l}\mathrm{Fi} \\
\mathrm{xe}\end{array}$ \\
\hline 100 & $\begin{array}{l}5 \\
5\end{array}$ & $\begin{array}{l}2 \\
3\end{array}$ & $\begin{array}{r}\mathrm{MB} \\
30\end{array}$ & $\begin{array}{l}\mathrm{Fe} \\
41\end{array}$ & $\begin{array}{l}\text { Non-c } \\
\text { ompos }\end{array}$ & $\begin{array}{l}\mathrm{Fi} \\
\mathrm{xe}\end{array}$ & $\begin{array}{l}\mathrm{Fi} \\
\mathrm{xe}\end{array}$ & & xe & & $\begin{array}{l}\mathrm{Fi} \\
\mathrm{xe}\end{array}$ & $\begin{array}{l}\mathrm{Fr} \\
\mathrm{ee}\end{array}$ & $\begin{array}{l}\mathrm{Fr} \\
\text { ee }\end{array}$ \\
\hline 105 & $\begin{array}{l}2 \\
3\end{array}$ & $\begin{array}{l}5 \\
7\end{array}$ & $\begin{array}{c}\mathrm{MB} \\
30\end{array}$ & $\begin{array}{l}\mathrm{Fe} \\
41\end{array}$ & $\begin{array}{l}\text { Non-c } \\
\text { ompos }\end{array}$ & $\begin{array}{l}\mathrm{Fi} \\
\mathrm{xe}\end{array}$ & xe & & $\mathrm{Fr}$ & $\begin{array}{c}\mathrm{Fi} \\
\mathrm{xe}\end{array}$ & $\begin{array}{l}\mathrm{Fi} \\
\mathrm{xe}\end{array}$ & & $\begin{array}{l}\mathrm{Fi} \\
\mathrm{xe}\end{array}$ \\
\hline 106 & $\begin{array}{l}5 \\
7\end{array}$ & $\begin{array}{l}3 \\
2 \\
\end{array}$ & $\begin{array}{r}\mathrm{MB} \\
30\end{array}$ & $\begin{array}{l}\mathrm{Fe} \\
41\end{array}$ & $\begin{array}{l}\text { Non-c } \\
\text { ompos }\end{array}$ & $\begin{array}{c}\mathrm{Fi} \\
\mathrm{xe} \\
\end{array}$ & $\mathrm{xe}$ & $\begin{array}{c}\mathrm{Fi} \\
\mathrm{xe}\end{array}$ & $\mathrm{F}$ & $\begin{array}{c}\mathrm{Fi} \\
\mathrm{xe}\end{array}$ & $\begin{aligned} x e \\
\text { Xe }\end{aligned}$ & $\begin{array}{l}\mathrm{Fr} \\
\mathrm{ee}\end{array}$ & $\mathrm{Fr}$ \\
\hline
\end{tabular}

Table7: List of Node Section Details with DOF

\section{Loads and load combinations}

Loads that are calculated manually are defined as shown in Figure 10.

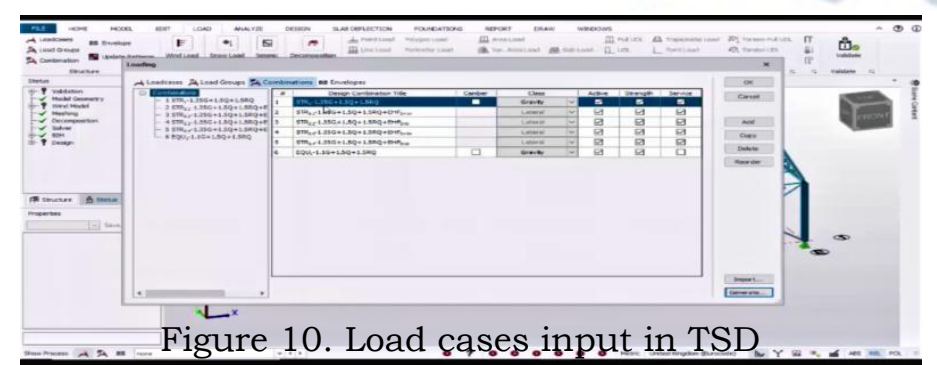

When applying wind loads to the structure, it is important to define which side of the structure is under pressure. Corners of the building are marked with letters A, B, C and D in the software. Thus, wind is acting perpendicularly on the sides $A B, C D$, $\mathrm{BC}$ and $\mathrm{DA}$ as shown in Figure 11

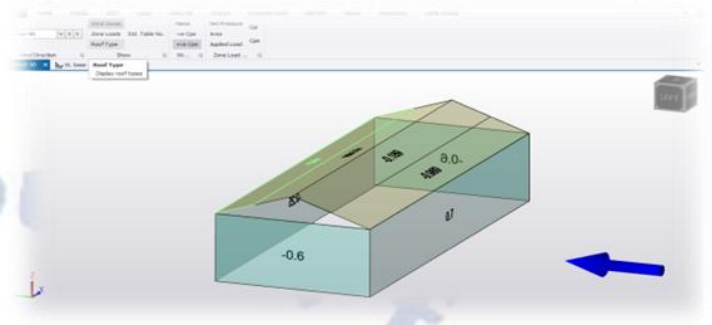

Figure 11. Designations of sides of the structure and pressure zones in TSD

Along wind forces on slender and wind sensitive structures and structural elements shall also be computed, for dynamic effects, using the Gust Factor or Gust Effectiveness Factor Method as defined in the standard. The structures shall be designed for the higher of the forces obtained from Gust Factor method and the Peak Wind Speed method.

Susceptibility of structures to a cross-wind forces, galloping, flutter, oval ling etc. should be examined and designed/detailed accordingly following the recommendations of IS:875(Part-3) and other relevant Indian standards.

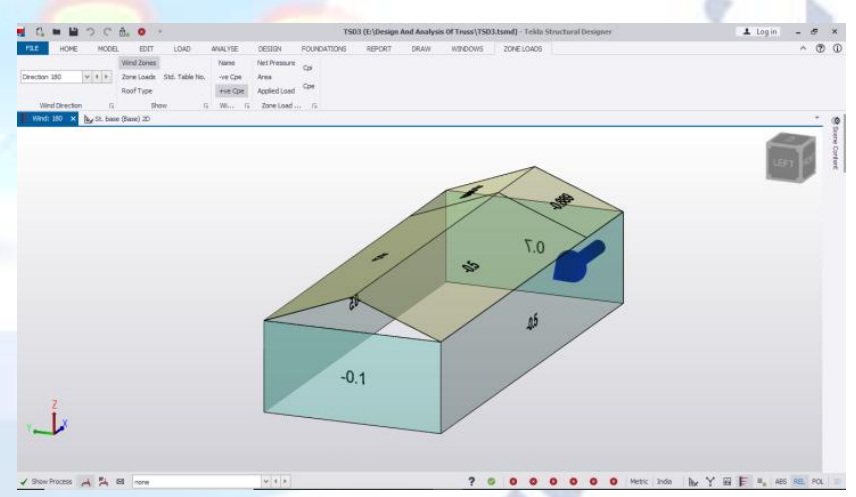

Figure 12. Designations of sides of the structure and pressure zones in TSD

Load combinations are used for structural analysis. A load combination input is shown in Figure 13 below. 


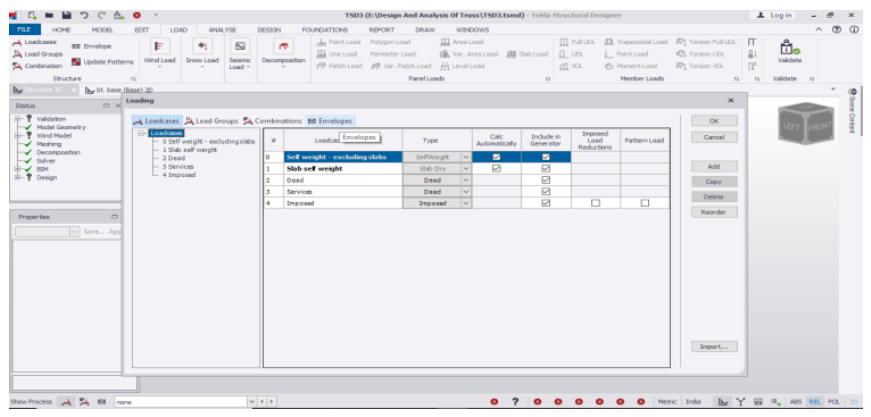

Figure 13. Load combinations input in Tekla structural designer

\section{Wind Load case Summary:}

All structures shall be designed for wind forces in accordance with IS: 875 (Part-3) and as specified in this Project. Along wind forces shall generally be computed by the Peak (i.e. 3 second gust) Wind speed method as defined in the standard. Since a structure obstructs the flow of air, a load acts normal to the exposed surface of the structure which is known as wind load. This is an important load on light weight structures, high rise buildings and towers and bridges. IS 875 (Part 3) : 1987 specifies the wind load to be considered for buildings and structures.

\begin{tabular}{|l|c|c|c|}
\hline \multirow{2}{*}{ Load Type } & \multicolumn{3}{c|}{ Force [kN] } \\
\cline { 2 - 4 } & $\mathbf{X}$ & $\mathbf{Y}$ & $\mathbf{Z}$ \\
\hline Member Loads & 0.00 & 0.00 & 0.00 \\
\hline Nodal Loads & 0.00 & 0.00 & 0.00 \\
\hline Total NHF Dir1 & 0.00 & 0.00 & 0.00 \\
\hline Total NHF Dir2 & 0.00 & 0.00 & 0.00 \\
\hline Decomposable Loads & 0.00 & 0.00 & 0.00 \\
\hline 1 Way Decomp Results & 0.00 & 0.00 & 0.00 \\
\hline 2 Way Decomp Results & 0.00 & 0.00 & 0.00 \\
\hline Total User Applied Load & 0.00 & 0.00 & 0.00 \\
\hline Total Load on Structure & 0.00 & 0.00 & 0.00 \\
\hline Total Reaction & 0.00 & 0.00 & 0.00 \\
\hline
\end{tabular}

Table 8: Wind load case Details

- $\quad$ wind 0,Cpi 0.2

\begin{tabular}{|l|c|c|c|}
\hline \multirow{2}{*}{ Load Type } & \multicolumn{3}{|c|}{ Force [kN] } \\
\cline { 2 - 4 } & $\mathbf{X}$ & $\mathbf{Y}$ & $\mathbf{Z}$ \\
\hline Member Loads & 0.00 & 0.00 & 0.00 \\
\hline
\end{tabular}

\begin{tabular}{|c|c|c|c|}
\hline \multirow[t]{2}{*}{ Load Type } & \multicolumn{3}{|c|}{ Force $[\mathbf{k N}]$} \\
\hline & $\mathbf{x}$ & $\mathbf{Y}$ & $\mathbf{Z}$ \\
\hline Nodal Loads & 0.00 & 0.00 & 0.00 \\
\hline Total NHF Dir1 & 0.00 & 0.00 & 0.00 \\
\hline Total NHF Dir2 & 0.00 & 0.00 & 0.00 \\
\hline Decomposable & 48.41 & 0.00 & -180.09 \\
\hline 1 Way Decomp & 0.00 & 0.00 & 0.00 \\
\hline 2 Way Decomp & 0.00 & 0.00 & 0.00 \\
\hline Total User Applied & 48.41 & 0.00 & -180.09 \\
\hline Total Load on & 48.41 & 0.00 & -180.09 \\
\hline Total Reac & -48.41 & 0.00 & -180.09 \\
\hline
\end{tabular}

Table 9: Wind load case Details (Wind 0,Cpi 0.2)

- $\quad$ Wind 90,Cpi -0.2

\begin{tabular}{|l|c|c|c|}
\hline \multirow{2}{*}{\multicolumn{1}{|c|}{ Load Type }} & \multicolumn{3}{c|}{ Force [kN] } \\
\cline { 2 - 4 } & $\mathbf{X}$ & $\mathbf{Y}$ & $\mathbf{Z}$ \\
\hline Member Loads & 0.00 & 0.00 & 0.00 \\
\hline Nodal Loads & 0.00 & 0.00 & 0.00 \\
\hline Total NHF Dir1 & 0.00 & 0.00 & 0.00 \\
\hline Total NHF Dir2 & 0.00 & 0.00 & 0.00 \\
\hline Decomposable Loads & 0.00 & 102.73 & -118.09 \\
\hline 1 Way Decomp Results & 0.00 & 0.00 & 0.00 \\
\hline 2 Way Decomp Results & 0.00 & 0.00 & 0.00 \\
\hline Total User Applied Load & 0.00 & 102.73 & -118.09 \\
\hline Total Load on Structure & 0.00 & 102.73 & -118.09 \\
\hline Total Reaction & 0.00 & -102.73 & -118.09 \\
\hline
\end{tabular}

Table 10: Wind load case Details (Wind 90,Cpi $-0.2)$

- $\quad$ Wind 90,Cpi 0.2

\begin{tabular}{|l|c|c|c|}
\hline \multirow{2}{*}{\multicolumn{1}{|c|}{ Load Type }} & \multicolumn{3}{c|}{ Force [kN] } \\
\cline { 2 - 4 } & $\mathbf{X}$ & $\mathbf{Y}$ & $\mathbf{Z}$ \\
\hline Member Loads & 0.00 & 0.00 & 0.00 \\
\hline Nodal Loads & 0.00 & 0.00 & 0.00 \\
\hline Total NHF Dir1 & 0.00 & 0.00 & 0.00 \\
\hline Total NHF Dir2 & 0.00 & 0.00 & 0.00 \\
\hline Decomposable Loads & 0.00 & 102.73 & -204.54 \\
\hline 1 Way Decomp Results & 0.00 & 0.00 & 0.00 \\
\hline 2 Way Decomp Results & 0.00 & 0.00 & 0.00 \\
\hline Total User Applied Load & 0.00 & 102.73 & -204.54 \\
\hline Total Load on Structure & 0.00 & 102.73 & -204.54 \\
\hline Total Reaction & 0.00 & -102.73 & -204.54 \\
\hline
\end{tabular}

Table 11: Wind load case Details (Wind 90,Cpi $0.2)$ 
- Wind 180, Cpi -0.2

\begin{tabular}{|l|c|c|c|}
\hline \multirow{1}{*}{ Load Type } & \multicolumn{3}{c|}{ Force $[\mathbf{k N}]$} \\
\cline { 2 - 4 } & $\mathbf{X}$ & $\mathbf{Y}$ & $\mathbf{Z}$ \\
\hline Member Loads & 0.00 & 0.00 & 0.00 \\
\hline Nodal Loads & 0.00 & 0.00 & 0.00 \\
\hline Total NHF Dir1 & 0.00 & 0.00 & 0.00 \\
\hline Total NHF Dir2 & 0.00 & 0.00 & 0.00 \\
\hline Decomposable Loads & -48.41 & 0.00 & -93.65 \\
\hline 1 Way Decomp Results & 0.00 & 0.00 & 0.00 \\
\hline 2 Way Decomp Results & 0.00 & 0.00 & 0.00 \\
\hline Total User Applied Load & -48.41 & 0.00 & -93.65 \\
\hline Total Load on Structure & -48.41 & 0.00 & -93.65 \\
\hline Total Reaction & 48.41 & 0.00 & -93.65 \\
\hline
\end{tabular}

Table 12: Wind load case Details (Wind 180, Cpi -0.2)

- Wind 180,Cpi 0.2

\begin{tabular}{|l|c|c|c|}
\hline \multirow{2}{*}{\multicolumn{1}{c|}{ Load Type }} & \multicolumn{3}{c|}{ Force $[\mathbf{k N}]$} \\
\cline { 2 - 4 } & $\mathbf{X}$ & $\mathbf{Y}$ & $\mathbf{Z}$ \\
\hline Member Loads & 0.00 & 0.00 & 0.00 \\
\hline Nodal Loads & 0.00 & 0.00 & 0.00 \\
\hline Total NHF Dir1 & 0.00 & 0.00 & 0.00 \\
\hline Total NHF Dir2 & 0.00 & 0.00 & 0.00 \\
\hline Decomposable Loads & -48.41 & 0.00 & -180.09 \\
\hline 1 Way Decomp Results & 0.00 & 0.00 & 0.00 \\
\hline 2 Way Decomp Results & 0.00 & 0.00 & 0.00 \\
\hline Total User Applied Load & -48.41 & 0.00 & -180.09 \\
\hline Total Load on Structure & -48.41 & 0.00 & -180.09 \\
\hline Total Reaction & 48.41 & 0.00 & -180.09 \\
\hline
\end{tabular}

Table 13: Wind load case Details (Wind 180,Cpi 0.2)

- Winds 270, Cpi -0.2

\begin{tabular}{|l|c|c|c|}
\hline \multirow{2}{*}{\multicolumn{1}{|c|}{ Load Type }} & \multicolumn{3}{|c|}{ Force [kN] } \\
\cline { 2 - 4 } & $\mathbf{X}$ & $\mathbf{Y}$ & $\mathbf{Z}$ \\
\hline Member Loads & 0.00 & 0.00 & 0.00 \\
\hline Nodal Loads & 0.00 & 0.00 & 0.00 \\
\hline Total NHF Dir1 & 0.00 & 0.00 & 0.00 \\
\hline Total NHF Dir2 & 0.00 & 0.00 & 0.00 \\
\hline Decomposable Loads & 0.00 & -102.73 & -118.09 \\
\hline 1 Way Decomp Results & 0.00 & 0.00 & 0.00 \\
\hline 2 Way Decomp Results & 0.00 & 0.00 & 0.00 \\
\hline Total User Applied Load & 0.00 & -102.73 & -118.09 \\
\hline Total Load on Structure & 0.00 & -102.73 & -118.09 \\
\hline Total Reaction & 0.00 & 102.73 & -118.09 \\
\hline
\end{tabular}

Table 14: Wind load case Details(Winds 270, Cpi -0.2)
- Wind 270,Cpi 0.2

\begin{tabular}{|l|r|c|c|}
\hline \multirow{2}{*}{ Load Type } & \multicolumn{3}{c|}{ Force [kN] } \\
\cline { 2 - 4 } & $\mathbf{X}$ & $\mathbf{Y}$ & $\mathbf{Z}$ \\
\hline Member Loads & 0.00 & 0.00 & 0.00 \\
\hline Nodal Loads & 0.00 & 0.00 & 0.00 \\
\hline Total NHF Dir1 & 0.00 & 0.00 & 0.00 \\
\hline Total NHF Dir2 & 0.00 & 0.00 & 0.00 \\
\hline Decomposable Loads & 0.00 & -102.73 & -204.54 \\
\hline 1 Way Decomp Results & 0.00 & 0.00 & 0.00 \\
\hline 2 Way Decomp Results & 0.00 & 0.00 & 0.00 \\
\hline Total User Applied Load & 0.00 & -102.73 & -204.54 \\
\hline Total Load on Structure & 0.00 & -102.73 & -204.54 \\
\hline Total Reaction & 0.00 & 102.73 & -204.54 \\
\hline
\end{tabular}

Table 15: Wind load case Details (Wind 270,Cpi $0.2)$

When the load cases and load combinations are defined, the loads may be assigned to the structure.

\section{Dead Load :}

Dead load means the self-weight of the structureor its components. This depends on the unit weight of materials used in the structure and the dimensions of the structure or its components. This is obtained by multiplying the volume of structure or its component with the unit weight. IS 875 (Part 1): 1987 gives the unit weights of various materials used in constructionDead loads shall include the weight of structure complete with finishes, fixtures and partitions and shall be taken as per IS: 875 (Part - 1).

\section{Load case Summary}

1 Self weight - excluding slabs

\begin{tabular}{|l|c|c|c|}
\hline \multirow{2}{*}{\multicolumn{1}{|c|}{ Load Type }} & \multicolumn{3}{|c|}{ Force [kN] } \\
\cline { 2 - 4 } & $\mathbf{X}$ & $\mathbf{Y}$ & $\mathbf{Z}$ \\
\hline Member Loads & 0.00 & 0.00 & 66.40 \\
\hline Nodal Loads & 0.00 & 0.00 & 9.88 \\
\hline Total NHF Dir1 & 0.30 & 0.00 & 0.00 \\
\hline Total NHF Dir2 & 0.00 & 0.30 & 0.00 \\
\hline Decomposable Loads & 0.00 & 0.00 & 0.00 \\
\hline 1 Way Decomp Results & 0.00 & 0.00 & 0.00 \\
\hline 2 Way Decomp Results & 0.00 & 0.00 & 0.00 \\
\hline Total User Applied Load & 0.00 & 0.00 & 76.28 \\
\hline Total Load on Structure & 0.00 & 0.00 & 76.28 \\
\hline Total Reaction & 0.00 & 0.00 & 76.28 \\
\hline
\end{tabular}

Table 16: Dead load case Details

2 Slab self-weight 


\begin{tabular}{|l|c|c|c|}
\hline \multirow{2}{*}{ Load Type } & \multicolumn{3}{c|}{ Force [kN] } \\
\cline { 2 - 4 } & $\mathbf{X}$ & $\mathbf{Y}$ & $\mathbf{Z}$ \\
\hline Member Loads & 0.00 & 0.00 & 0.00 \\
\hline Nodal Loads & 0.00 & 0.00 & 0.00 \\
\hline Total NHF Dir1 & 0.00 & 0.00 & 0.00 \\
\hline Total NHF Dir2 & 0.00 & 0.00 & 0.00 \\
\hline Decomposable Loads & 0.00 & 0.00 & 0.00 \\
\hline 1 Way Decomp Results & 0.00 & 0.00 & 0.00 \\
\hline 2 Way Decomp Results & 0.00 & 0.00 & 0.00 \\
\hline Total User Applied Load & 0.00 & 0.00 & 0.00 \\
\hline Total Load on Structure & 0.00 & 0.00 & 0.00 \\
\hline Total Reaction & 0.00 & 0.00 & 0.00 \\
\hline
\end{tabular}

Table 17: Dead load case Details

\begin{tabular}{|l|c|c|c|}
\hline \multirow{2}{*}{ Load Type } & \multicolumn{3}{|c|}{ Force [kN] } \\
\cline { 2 - 4 } & $\mathbf{X}$ & $\mathbf{Y}$ & $\mathbf{Z}$ \\
\hline Member Loads & 0.00 & 0.00 & 0.00 \\
\hline Nodal Loads & 0.00 & 0.00 & 0.00 \\
\hline Total NHF Dir1 & 3.01 & 0.00 & 0.00 \\
\hline Total NHF Dir2 & 0.00 & 3.01 & 0.00 \\
\hline Decomposable Loads & 0.00 & 0.00 & 602.99 \\
\hline 1 Way Decomp Results & 0.00 & 0.00 & 602.99 \\
\hline 2 Way Decomp Results & 0.00 & 0.00 & 0.00 \\
\hline Total User Applied Load & 0.00 & 0.00 & 602.99 \\
\hline Total Load on Structure & 0.00 & 0.00 & 602.99 \\
\hline Total Reaction & 0.00 & 0.00 & 602.99 \\
\hline
\end{tabular}

Table 18: Dead load case Details

\section{Imposed Loads}

This is the load due to intended use oroccupancy which may be stationary or moving. It includes the load due to impact or vibration. In buildings, it includes the weight of the occupants, the various things and materials keep on the floors Imposed loads in different areas shall include live, erection, operation and maintenance loads. Equipment loads (which constitute all loads of equipment to besupported on the building frame) are not included in the imposed loads furnished Se below and shall be considered in addition to imposed loads. Figure 14 shows the Roof imposed load applied to the structure.

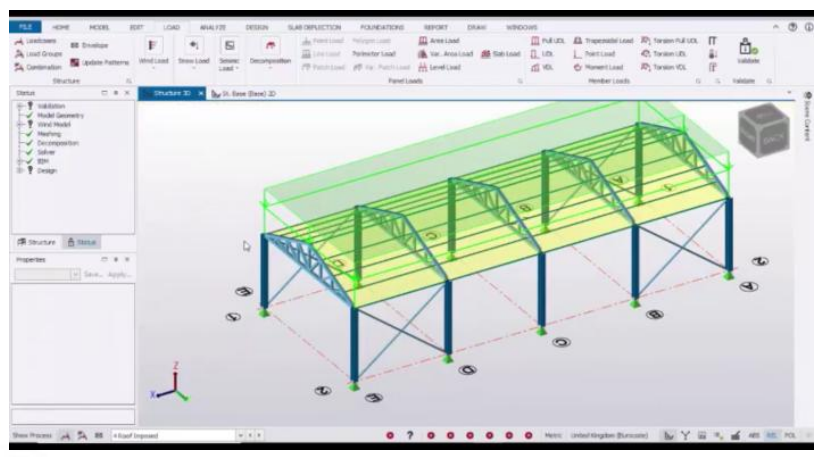

Figure 14. Roof imposed load assigned to the structure in Tekla structural designer

\begin{tabular}{|l|c|c|c|}
\hline \multicolumn{1}{|c|}{ Load Type } & \multicolumn{3}{c|}{ Force [kN] } \\
\cline { 2 - 4 } & $\mathbf{X}$ & $\mathbf{Y}$ & $\mathbf{Z}$ \\
\hline Member Loads & 0.00 & 0.00 & 0.00 \\
\hline Nodal Loads & 0.00 & 0.00 & 0.00 \\
\hline Total NHF Dir1 & 3.01 & 0.00 & 0.00 \\
\hline Total NHF Dir2 & 0.00 & 3.01 & 0.00 \\
\hline $\begin{array}{l}\text { Decomposable Loads } \\
\text { 1 Way Decomp }\end{array}$ & 0.00 & 0.00 & 602.99 \\
\hline $\begin{array}{l}\text { 1 Wesults } \\
\text { 2 Way Decomp } \\
\text { Results }\end{array}$ & 0.00 & 0.00 & 602.99 \\
\hline
\end{tabular}

table19:Imposed load sumary

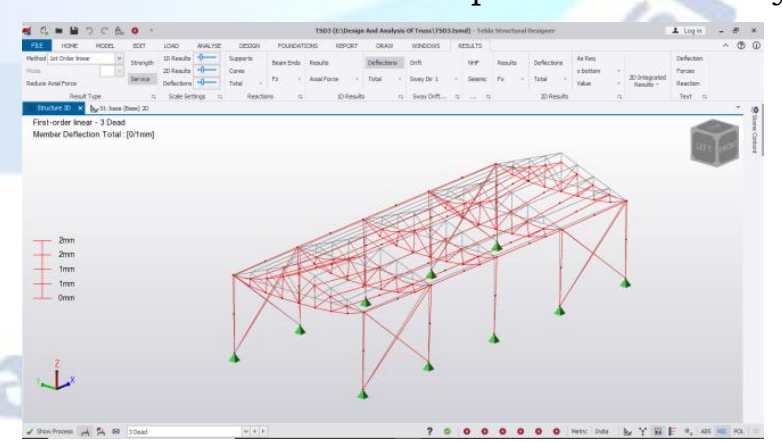

Figure 15: Shows total Member Deflections 


\section{Sway}

First-order linear

\begin{tabular}{|c|c|c|c|c|c|c|c|c|c|}
\hline & $\begin{array}{l}\text { Combin } \\
\text { ation }\end{array}$ & & & $\begin{array}{l}\text { Combin } \\
\text { ation }\end{array}$ & & & $\begin{array}{l}\text { Com } \\
\text { binat }\end{array}$ & & S \\
\hline p & $1.5 \mathrm{~L}+1$ & & \begin{tabular}{|l|}
40 \\
1.1 \\
78
\end{tabular} & $\begin{array}{l}\mathrm{LS}_{2}-1.5 \mathrm{D} \\
+1.5 \mathrm{~L}+1\end{array}$ & 1 & $\begin{array}{l}22 \\
.5 \\
80\end{array}$ & IVe & 0 & \\
\hline & & 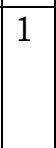 & $\begin{array}{l}.1 \\
8\end{array}$ & & 1 & $\begin{array}{l}22 \\
.5 \\
80\end{array}$ & $c t$ & 0 & \\
\hline & & 1 & $\begin{array}{l}.1 \\
8\end{array}$ & & 1 & $\begin{array}{l}22 \\
.5 \\
80\end{array}$ & $c t$ & 0 & \\
\hline & & 1 & & & 1 & $\begin{array}{l}22 \\
.5 \\
80\end{array}$ & $i$ & 0 & \\
\hline & & 1 & & & 1 & $\begin{array}{l}22 \\
.6 \\
88\end{array}$ & ive & 0 & \\
\hline & & 1 & $\begin{array}{l}1.5 \\
96\end{array}$ & & 1 & $\begin{array}{l}22 \\
.6 \\
88\end{array}$ & & 0 & \\
\hline & & 1 & \begin{tabular}{|l|}
38 \\
1.5 \\
96
\end{tabular} & $\mathrm{~L}+1$ & 1 & $\begin{array}{l}22 \\
.6 \\
88\end{array}$ & $\begin{array}{l}\text { Effect } \\
\text { ive }\end{array}$ & 0 & Ss \\
\hline & $\begin{array}{l}\text { al) } \\
5 \mathrm{D} \\
-1 .\end{array}$ & 1 & \begin{tabular}{|l|}
38 \\
1.5 \\
96
\end{tabular} & nal) & 1 & $\begin{array}{l}22 \\
.6 \\
88\end{array}$ & \begin{tabular}{|l}
30 \\
Effect \\
ive
\end{tabular} & 17 & $\cdot \begin{array}{l}7 \\
P\end{array}$ \\
\hline
\end{tabular}

Table 20: sway details

\section{Analysis of biaxial bending andtorsion}

Methods of analyzing biaxial bending and torsion are generally limited to first-order elastic analyses, and many of these omit the strengthening effects of warping torsion in open section members. Further difficulties arise when second-order effects must be allowed for.

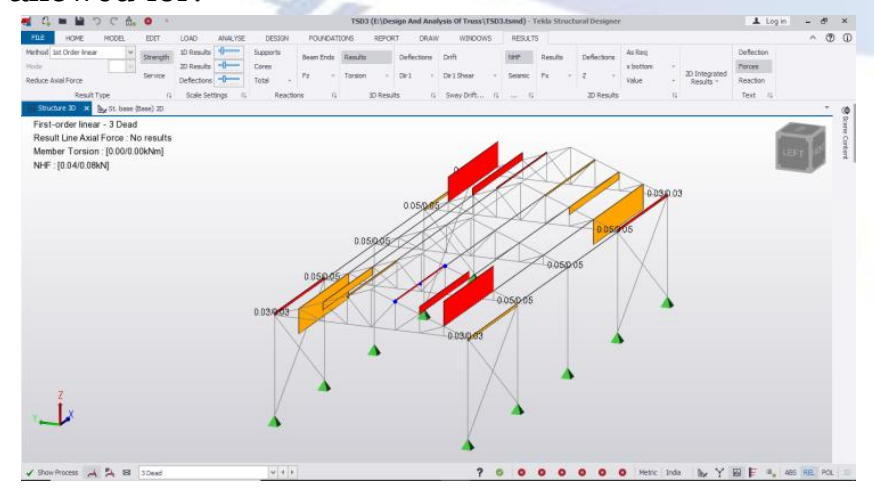

Figure 16: First order Linear Member Torsion
Column Design Summary

Static

\begin{tabular}{|c|c|c|c|c|c|c|c|}
\hline $\begin{array}{c}\text { Memb } \\
\text { er } \\
\text { Refere }\end{array}$ & $\begin{array}{c}\text { Grou } \\
\text { p Ref. }\end{array}$ & $\begin{array}{c}\text { Sta } \\
\text { ck }\end{array}$ & $\begin{array}{c}\text { Secti } \\
\text { on }\end{array}$ & $\begin{array}{c}\text { Gra } \\
\text { de }\end{array}$ & $\begin{array}{l}\text { Leng } \\
\text { th } \\
{[\mathrm{m}]}\end{array}$ & $\begin{array}{c}\text { Utiliza } \\
\text { tion }\end{array}$ & us \\
\hline $\begin{array}{c}\text { Span } 1 \\
\text { Lh }\end{array}$ & \begin{tabular}{|c|}
$\mathrm{PF}$ \\
$\mathrm{A} / 1-\mathrm{A}$
\end{tabular} & 1 & $\begin{array}{c}\mathrm{SC} \\
250\end{array}$ & \begin{tabular}{c|}
$\mathrm{Fe}$ \\
410
\end{tabular} & \begin{tabular}{|c}
4.00 \\
0
\end{tabular} & .667 & $\mathrm{~Pa}$ \\
\hline $\begin{array}{c}\text { Span 1 } \\
\text { Rh }\end{array}$ & $\begin{array}{c}\mathrm{PF} \\
\mathrm{A} / 1-\mathrm{A}\end{array}$ & 1 & $\begin{array}{c}\mathrm{SC} \\
250\end{array}$ & $\begin{array}{c}\mathrm{Fe} \\
410\end{array}$ & \begin{tabular}{|c|}
4.00 \\
0
\end{tabular} & .667 & $\mathrm{~Pa}$ \\
\hline $\begin{array}{c}\text { Span } 1 \\
\text { Lh }\end{array}$ & $\begin{array}{c}\mathrm{PF} \\
\mathrm{B} / 1-\end{array}$ & 1 & $\begin{array}{l}\text { HB } \\
450\end{array}$ & \begin{tabular}{c|}
$\mathrm{Fe}$ \\
410
\end{tabular} & $\begin{array}{c}4.00 \\
0\end{array}$ & .934 & 10 \\
\hline $\begin{array}{c}\text { Span } 1 \\
\text { Rh }\end{array}$ & $\begin{array}{c}\mathrm{PF} \\
\mathrm{B} / 1-\end{array}$ & 1 & $\begin{array}{l}\text { HB } \\
450\end{array}$ & $\begin{array}{c}\mathrm{Fe} \\
410\end{array}$ & $\begin{array}{c}4.00 \\
0\end{array}$ & 0.934 & $\mathrm{~Pa}$ \\
\hline $\begin{array}{c}\text { Span } 1 \\
\text { Lh }\end{array}$ & $\begin{array}{c}\mathrm{PF} \\
\mathrm{C} / 1-\end{array}$ & 1 & $\begin{array}{l}\text { HB } \\
450\end{array}$ & $\begin{array}{c}\mathrm{Fe} \\
410\end{array}$ & $\begin{array}{c}4.00 \\
0\end{array}$ & 34 & $\mathrm{~Pa}$ \\
\hline $\begin{array}{c}\text { Span } 1 \\
\mathrm{Rh}\end{array}$ & \begin{tabular}{|c|}
$\mathrm{PF}$ \\
$\mathrm{C} / 1-$
\end{tabular} & 1 & $\begin{array}{l}\mathrm{HB} \\
450\end{array}$ & \begin{tabular}{c|}
$\mathrm{Fe}$ \\
410
\end{tabular} & \begin{tabular}{|c|}
4.00 \\
0
\end{tabular} & 934 & $\mathrm{~Pa}$ \\
\hline $\begin{array}{c}\text { Span } 1 \\
\text { Lh }\end{array}$ & $\begin{array}{c}\mathrm{PF} \\
\mathrm{D} / 1-\end{array}$ & 1 & $\begin{array}{c}\mathrm{SC} \\
250\end{array}$ & $\begin{array}{c}\mathrm{Fe} \\
410\end{array}$ & \begin{tabular}{|c|}
4.00 \\
0
\end{tabular} & 0.667 & $\mathrm{~Pa}$ \\
\hline $\begin{array}{c}\text { Span } 1 \\
\text { Rh }\end{array}$ & $\begin{array}{c}\mathrm{PF} \\
\mathrm{D} / 1-\end{array}$ & 1 & $\begin{array}{c}\text { SC } \\
250\end{array}$ & \begin{tabular}{c|}
$\mathrm{Fe}$ \\
410
\end{tabular} & \begin{tabular}{|c|}
4.00 \\
0
\end{tabular} & 667 & $\mathrm{~Pa}$ \\
\hline
\end{tabular}

Table 20: Column Design summary

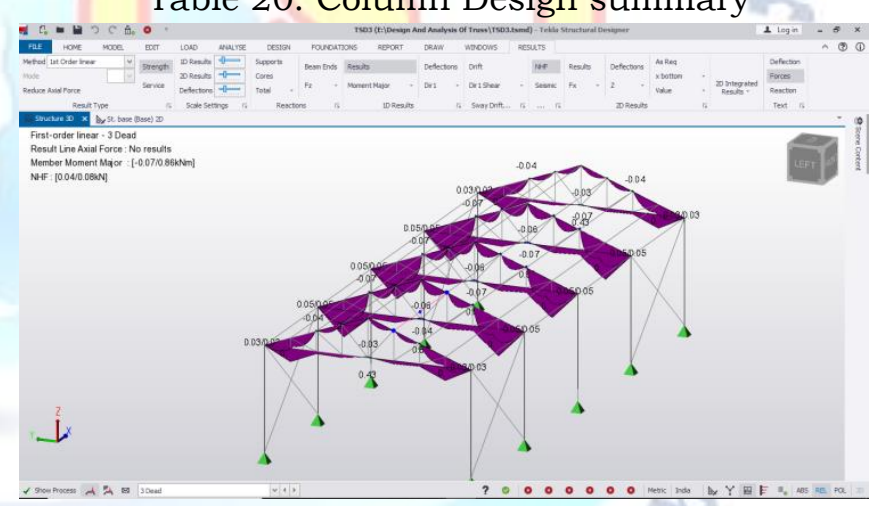

Figure 17: Shows First Order Linear Member Moment Major Axis

Beam Design Summary

Static

\begin{tabular}{|c|c|c|c|c|c|c|c|c|}
\hline Membe & Gro & Sp & Sec & Gr & Len & No. & Utiliz & Sta \\
\hline Span 1 & PF & 1 & MB & Fe & 4.0 & & 0.905 & $\checkmark$ \\
\hline Span 1 & PF & 1 & MB & Fe & 4.0 & & 0.905 & $\checkmark$ \\
\hline Span 1 & PF & 1 & HB & Fe & 4.0 & & 0.827 & $\checkmark$ \\
\hline Span 1 & PF & 1 & HB & Fe & 4.0 & & 0.827 & $\checkmark$ \\
\hline Span 1 & PF & 1 & HB & Fe & 4.0 & & 0.827 & $\checkmark$ \\
\hline Span 1 & PF & 1 & HB & Fe & 4.0 & & 0.827 & $\checkmark$ \\
\hline Span 1 & PF & 1 & MB & Fe & 4.0 & & 0.905 & $\checkmark$ \\
\hline Span 1 & PF & 1 & MB & Fe & 4.0 & & 0.905 & $\checkmark$ \\
\hline SB & SBR & 1 & MB & Fe & 5.0 & & 0.928 & $\checkmark$ \\
\hline SB & SBR & 1 & MB & Fe & 5.0 & & 0.704 & $\checkmark$ \\
\hline SB & SBR & 1 & MB & Fe & 5.0 & & 0.928 & $\checkmark$ \\
\hline SB & SBR & 1 & MB & Fe & 5.0 & & 0.978 & $\checkmark$ \\
\hline SB & SBR & 1 & MB & Fe & 5.0 & & 0.704 & $\checkmark$ \\
\hline
\end{tabular}




\begin{tabular}{|c|c|c|c|c|c|c|c|c|}
\hline Membe & Gro & Sp & Sec & Gr & Len & No. & Utiliz & Sta \\
\hline SB & SBR & 1 & MB & Fe & 5.0 & & 0.978 & $\checkmark$ \\
\hline SB & SBR & 1 & MB & Fe & 5.0 & & 0.928 & $\checkmark$ \\
\hline SB & SBR & 1 & MB & Fe & 5.0 & & 0.704 & $\checkmark$ \\
\hline SB & SBR & 1 & MB & Fe & 5.0 & & 0.928 & $\checkmark$ \\
\hline
\end{tabular}

Table 21: Beam design summary

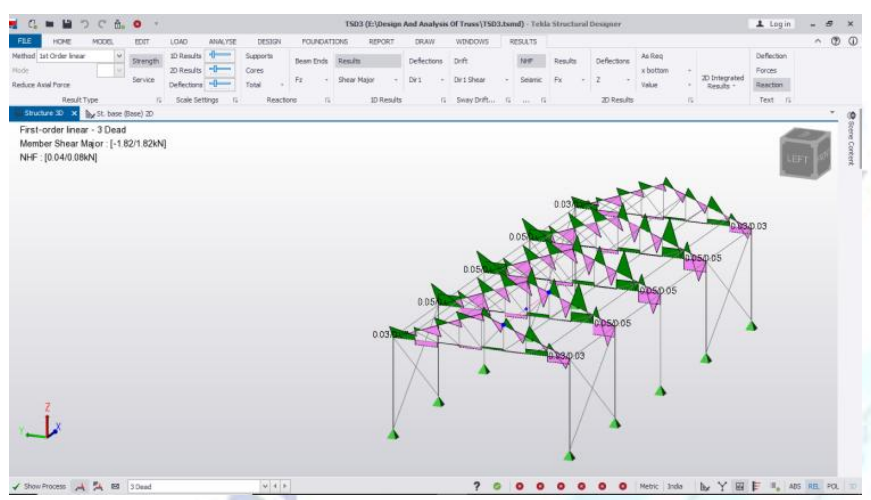

Figure 18: Shows First Order Linear Member Shear Major

Brace Design Summary

Static

\begin{tabular}{|c|c|c|c|c|c|c|c|}
\hline $\begin{array}{l}\text { Member } \\
\text { Reference }\end{array}$ & $\begin{array}{c}\text { Gro } \\
\text { up } \\
\text { Ref }\end{array}$ & $\begin{array}{l}\text { Sp } \\
\text { an }\end{array}$ & $\begin{array}{c}\text { Sect } \\
\text { ion }\end{array}$ & $\begin{array}{c}\text { Gra } \\
\text { de }\end{array}$ & $\begin{array}{l}\text { Len } \\
\text { gth } \\
\text { [m] }\end{array}$ & $\begin{array}{c}\text { Utiliza } \\
\text { tion }\end{array}$ & \\
\hline 1 & & 1 & OOL & & 40 & 0.908 & \\
\hline SB & $3 r$ & 1 & DOL & & 5.40 & .908 & \\
\hline SBR & $\mathrm{Br}$ & 1 & $100 \mathrm{~L}$ & $\mathrm{e}$ & 2.41 & 0.655 & \\
\hline $\mathrm{SE}$ & $\mathrm{Br}$ & 1 & OOL & e & 5.41 & 0.910 & \\
\hline SBF & $\mathrm{Br}$ & 1 & DOL & $\mathrm{Fe}$ & 6.41 & 10 & \\
\hline & $3 n$ & 1 & OOL & $\mathrm{Fe}$ & 41 & 0.655 & \\
\hline $\mathrm{SB}$ & $\mathrm{SBr}$ & 1 & DOL & $\mathrm{re}$ & 6.40 & 0.900 & \\
\hline$\frac{\text { Shad }}{\text { RB }}$ & $3 r$ & 1 & DOL & $\mathrm{Fe}$ & 6.40 & & \\
\hline $\begin{array}{l}\text { Dand } \\
\text { Sn }\end{array}$ & $\mathrm{Br}$ & 1 & & $\mathrm{Fe}$ & 6.40 & & \\
\hline مחת & & & & & & & \\
\hline & $3 r$ & 1 & DOL & $\mathrm{Fe}$ & 6.40 & 908 & \\
\hline SBR & $3 r$ & 1 & $100 \mathrm{~L}$ & $\mathrm{Fe}$ & 6.41 & & \\
\hline תות & & & & & & & \\
\hline SE & $\mathrm{SBr}$ & 1 & $100 \mathrm{~L}$ & e & 6.41 & 0.910 & \\
\hline & $3 r$ & 1 & $100 \mathrm{~L}$ & $\mathrm{Fe}$ & 6.40 & 0.908 & \\
\hline SB & $3 r$ & 1 & 0 & $\mathrm{Fe}$ & 6.40 & & \\
\hline $\begin{array}{l}\text { Rncol/1 } \\
\text { SBRR }\end{array}$ & $\begin{array}{l}\text { D1 } \\
\text { SBr }\end{array}$ & 1 & & & 6.41 & & \\
\hline & & 1 & 1 & & & & \\
\hline & & 1 & 100 & & 6.41 & 0.65 & \\
\hline
\end{tabular}

Table 22: Brace design summary

\section{Summary of Analysis:}

According to the AISC loads and design criteria, the Steel Structure of Truss type steel building is structurally analyzed with the help of FEM software TEKLA SOFTWARE. According to the analysis following things can be summarized:

$>$ All the Secondary beams arepassed.

Most of the Main beams are passed. Main beams $\&$ Bracing between grid 2 to 4 and $\mathrm{B}$ to $\mathrm{E}$ show utilization ratio 1.03 , which can be assumed as passed. But we will do further analysis in next step to make them under1.0.

D Some columns of base floor are in critical condition. Some of the columns are in critical condition due to lack of lateral bracing in thesystem.

$>$ Lateral defection is too high which is close to 36 inch (maximum) which is unacceptable. Again this occurs due to improper lateral structuralsystem.

\section{$>$ Roof Rafters arepassed.}

$>$ Some columns are in criticalcondition.

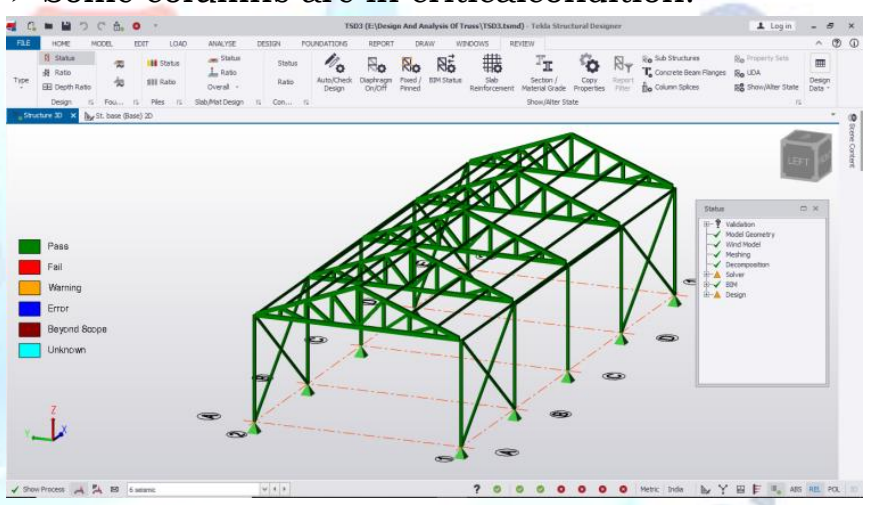

Figure 19: Shows Passed Truss Structure in Tekla Structural Designer

\section{CONCLUSION}

The aim of the thesis was to describe the process of structural analysis and design using the example of truss type steel building.

As the result of the project work, loads and load combinations were calculated, the structure was analyzed for stability, the joints were checked for resistance and finally, production drawings were made for further assembly. All the steps of the projects involved software such as TEKLA STRUCTURAL DESIGNER for structural analysis, Tekla Structures for modeling a 3D structure and later making production drawings and Mathcad for load calculations. Even though the sizes of the structural members were kept unchanged, possible modifications were discussed for the theoretical optimization of the structure. 
Hopefully, this thesis will serve as an example to those who would like to get a general idea of how structural analysis and design can be performed with the help of modern software. We emphasize more on quality in design. We have our own licensed Software like STAAD- Pro, ETABS, TEKLA, SAP, Quick Series, which are widely used in our design section. We always try to procure latest design manuals for our designers. We have latest Steel Construction Manuals: AISC Steel Construction manual Vol-13, MBMA Metal Building System Manual, Edition 2002, AISI Structural Welding Code - Steel, Edition: 2006.

Most of the structural engineers use ETABS or Staad Pro for analysis and design purpose. Further knowledge of more detailed software is a must for later run. Moreover one should always have a clear concept of theoretical knowledge prior to operating software. Regular updates of codes and conduct should be kept in mind while designing. For this purpose, attending national and international seminars on Structural Engineering is required. Last but not the least, one should always renew his/her active membership for AISC (American Institute of Steel Construction).

\section{REFERENCES}

[1] www.google.com

[2] www.wikipedia.com

[3] AISC (2011). Specification for Structural Steel Buildings. American Institute of Steel Construction, Chicago.

[4] BSI (2000). BS5950: Part 1:2000, Structural Use of Steelwork in Building, Part 1, Code of Practice for Design in Simple and Continuous Construction: Hot Rolled Sections. British Standards Institution,London.

[5] BSI (2005). Euro code 3: Design of Steel Structures: Part 1.1 General Rules and Rules for Buildings, BS EN 19931-1. British Standards Institution,London.

[6] Chan, S.L. (1989). "Inelastic post-buckling analysis of tubular beam-columns and frames". Journal of Engineering Structures, Vol. 11, pp.23-30.

[7] Engineering Structures, Vol. 22, No. 3, pp246-57.

[8] Chen, W.F. and Kim, S.E. (1997). LRFD Steel Design Using Advanced Analysis. CRC Press, Boca Raton, Florida.

[9] Clarke, M.J. (1994). "Plastic-zone analysis of frames". Advanced Analysis of Steel Frames: Theory, Software, and Applications. Chen, WF, and Toma, S, eds, CRC Press, Inc., Boca Raton, Florida, 1994; Chapter 6; pp.259-319.

[10] "Connection Performance for Seismic Design of Steel Moment Frames"byCharlesW.Roeder. Journal of Structural Engineering, 2002 ISSN:0733-9445.

[11] Comparision of structural modelling in open BIM projects by ValeriiaLobanovaDegreeProgramme in Civil and Construction Engineering Saimaa University of Applied Sciences Technology, Lappeenranta Bachelor's Thesis 2017. 9. Structural BIM modelling using TEKLA structures by AmanOli . Focus on a Modelling Process of an Office building. Helsinki Metropolia University of Applied Sciences. Apr 2017.
[12] N. Lingeshwaran K. Naga SaiGopalAnalysis and Design of $\mathrm{G}+5$ residential building by using E-TABS. International Journal of Civil Engineering and Technology (IJCIET) Volume 8, Issue 4, April 2017.

[13] S.A. Raji, A.A. Bello, Analysis of a Six Storey Steel Frame Structure using Autodesk Revit, Staadpro and SAP2000 Journal of Multidisciplinary Engineering Science and Technology (JMEST) ISSN: 2458-9403 Vol. 4 Issue 4, April $-2017$.

[14] AziziNaserabadAlifaz, Ghasemi Mohammad Reza "Evaluation of Beam-Flange (BF) Bolts on Behaviour of New BBCC Connection with Preferred Support in Modularized Prefabricated Steel Structures" journal of steel structures and construction 2018.

[15] Dr. P. D. Hiwase, Miss. Aditi Joshi, Mr.AakashKeshariya, Comparison between Manual and Software Approach towards Design of Structural Elements. The International Journal of Engineering and Science (IJES) ISSN (e): 2319 1813 ISSN (p): 23-19 - 1805Pages PP 54-56, 2018.

[16] K. Prabinkumar, D.Sunny Prakash "Planning Analysis and Design of Industrial Building Using STAAD PRO" International journal of pure and applied mathematics 2018.

[17] Vikrant Trivedi, SumitPahwa Wind Analysis and Design of G+11 Storied Building Using STAAD-Pro. International Research Journal of Engineering and Technology (IRJET) e-ISSN: 2395-0056 Volume: 05 Issue: 03 | Mar-2018

[18] M.A. Qureshi, NidhiBhavsar Comparison between Manual calculation and Software calculation of G+5 Building Using Staad pro. International Research Journal of Engineering and Technology (IRJET) e-ISSN: 2395-0056 Volume: 05, Issue: 05 May-2018.

[19] General construction in steel -IS:800-2007.

[20] "Design of Steel Structures" by S.K.Duggal.

[21] Manual design as per Indian code book IS 800:2007, and IS 875 for various loads.

[22] Design of steel structures by Dr. Ramchandra, Ph.D, degree in Civil Engineering from the University of Roorkey in 1984.

[23] Course on Design of Steel Structures Prof.DamodarMaity, Department of Civil Engineering Indian Institute of Technology Kharagpur 